\title{
MACHINE LEARNING-BASED ESTIMATION AND GOODNESS-OF-FIT FOR LARGE-SCALE CONFIRMATORY ITEM FACTOR ANALYSIS
}

\author{
Christopher J. Urban
}

A thesis submitted to the faculty of the University of North Carolina at Chapel Hill in partial fulfillment of the requirements for the degree of Master of Arts in the Department of Psychology and Neuroscience.

Chapel Hill

2021

Approved by:

Daniel J. Bauer

Kathleen M. Gates

Oscar Gonzalez

Marc Niethammer 
(C) 2021

Christopher J. Urban

ALL RIGHTS RESERVED 


\begin{abstract}
CHRISTOPHER J. URBAN: Machine Learning-Based Estimation and Goodness-of-Fit for Large-Scale Confirmatory Item Factor Analysis.

(Under the direction of Daniel J. Bauer)
\end{abstract}

I investigate novel parameter estimation and goodness-of-fit (GOF) assessment methods for large-scale confirmatory item factor analysis (IFA) with many respondents, items, and latent factors. For parameter estimation, I extend Urban and Bauer's (2021) deep learning algorithm for exploratory IFA to the confirmatory setting by showing how to handle user-defined constraints on loadings and factor correlations. For GOF assessment, I explore new simulation-based tests and indices. In particular, I consider extensions of the classifier two-sample test (C2ST), a method that tests whether a machine learning classifier can distinguish between observed data and synthetic data sampled from a fitted IFA model. The C2ST provides a flexible framework that integrates overall model fit, piece-wise fit, and person fit. Proposed extensions include a C2ST-based test of approximate fit in which the user specifies what percentage of observed data can be distinguished from synthetic data as well as a C2ST-based relative fit index that is similar in spirit to the relative fit indices used in structural equation modeling. Via simulation studies, I first show that the confirmatory extension of Urban and Bauer's (2021) algorithm produces more accurate parameter estimates as the sample size increases and obtains comparable estimates to a state-of-the-art confirmatory IFA estimation procedure in less time. I next show that the C2ST-based test of approximate fit controls the empirical type I error rate and detects when the number of latent factors is misspecified. Finally, I empirically investigate how the sampling distribution of the C2ST-based relative fit index depends on the sample size. 


\section{TABLE OF CONTENTS}

LIST OF TABLES $\ldots \ldots \ldots \ldots \ldots \ldots \ldots \ldots \ldots \ldots \ldots \ldots \ldots$ vi

LIST OF FIGURES $\ldots \ldots \ldots \ldots \ldots \ldots \ldots \ldots$ vii

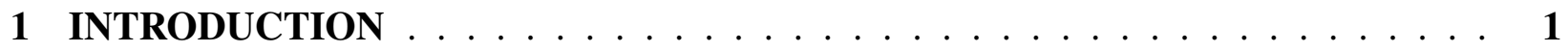

2 VARIATIONAL METHODS FOR PARAMETER ESTIMATION $\ldots \ldots \ldots \ldots$. . . 7

2.1 A Model for Confirmatory IFA . . . . . . . . . . . . . . . . . . 7

2.2 Variational Lower Bound $\ldots \ldots \ldots$. . . . . . . . . . . . . . . . . 9

2.3 Amortized VI . . . . . . . . . . . . . . . . . . . . 10

2.4 Importance-Weighted VI . . . . . . . . . . . . . . . . . . . . 13

2.5 Handling User-Defined Constraints . . . . . . . . . . . . . . . . . . . 15

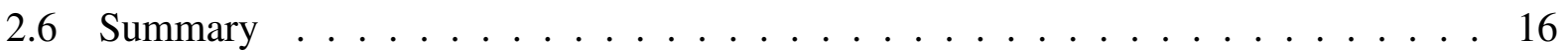

3 CLASSIFIER TWO-SAMPLE TESTS FOR GOODNESS-OF-FIT ASSESSMENT 17

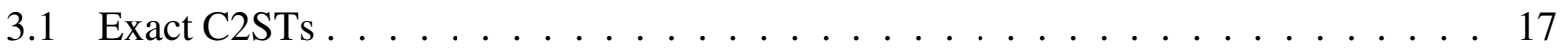

3.2 Approximate C2STs . . . . . . . . . . . . . . . . . . . . . . . . . . . . . 19

3.3 C2ST-Based Relative Fit Index . . . . . . . . . . . . . . . . . . . . 22

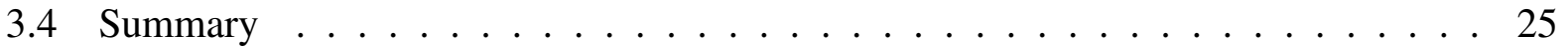

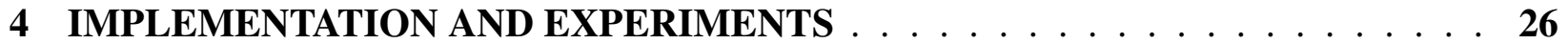

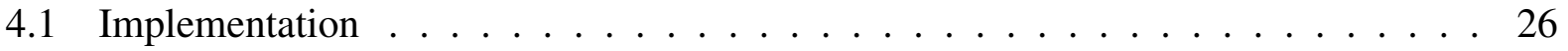

4.2 Empirical Example . . . . . . . . . . . . . . . . . . . 28

$4.2 .1 \quad$ A Five-Factor Model . . . . . . . . . . . . . . . . . . . . . . . 28 
4.2.2 A Seven-Factor Model . . . . . . . . . . . . . . . . . . . 33

4.3 Simulation Studies $\ldots \ldots \ldots \ldots$. . . . . . . . . . . . . . . 34

4.3 .1 Evaluating I-WAVE $\ldots \ldots \ldots \ldots$

4.3.2 Comparing I-WAVE to MH-RM . . . . . . . . . . . . . . 35

4.3.3 Evaluating Approximate C2STs in a Non-IFA Setting . . . . . . . . . . 38

4.3.4 Evaluating C2STs and C2ST-RFIs in a Confirmatory IFA Setting . . . . . . 41

5 DISCUSSION $\ldots \ldots \ldots \ldots \ldots \ldots \ldots \ldots \ldots \ldots$

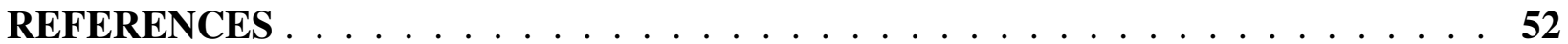




\section{LIST OF TABLES}

4.1 Factor Loadings for IPIP-FFM Data Set . . . . . . . . . . . . . . . . . . . . 29

4.2 Factor Correlations for IPIP-FFM Data Set . . . . . . . . . . . . . . . . . 30

4.3 Classifier Two-Sample Test Results for IPIP-FFM Data Set . . . . . . . . . . . . . . . 31

4.4 Wordings for Five Poorest Fitting IPIP-FFM Items _ . . . . . . . . . . . . . . . . 33 


\section{LIST OF FIGURES}

2.1 Schematic diagram of a variational autoencoder for confirmatory item factor analysis with $J=6$ items, $K=2$ categories per item, $P=2$ factors, $S=1$ Monte Carlo sample from the approximate latent variable posterior, and an inference model consisting of a neural network with a single hidden layer. Each factor loads on three items. The reparameterization of $\mathbf{z}$ is not illustrated for simplicity. $\mathrm{LV}=$ latent variable, $\mathrm{NN}=$ neural network. . . . . 13

4.1 Permutation importances for each IPIP-FFM item across 10 random starts. Items are grouped by their corresponding factor. Bar heights indicate medians, while error bars indicate $25 \%$ and $75 \%$ quantiles. Hatched bars indicate the five poorest fitting items for the five-factor model. EXT $=$ extraversion, EST $=$ emotional stability, AGR = agreeableness, $\mathrm{CON}=$ conscientiousness, $\mathrm{OPN}=$ openness. $\ldots \ldots \ldots \ldots \ldots \ldots \ldots \ldots \ldots$

4.2 Parameter bias for the importance-weighted amortized variational estimator (I-WAVE). Three settings for the number of importance-weighted (IW) samples are compared. . . . . 36

4.3 Parameter mean squared error (MSE) for I-WAVE. . . . . . . . . . . . . . 37

4.4 Fitting times for I-WAVE. Markers indicate medians, while error bars indicate $25 \%$

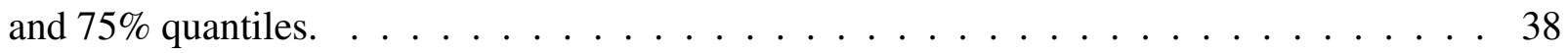

4.5 MSE for I-WAVE and the marginal maximum likelihood estimator. MH-RM = Metropolis-

Hastings Robbins-Monro. . . . . . . . . . . . . . . . . . . . . . . . . . . 39

4.6 Fitting times for I-WAVE and the marginal maximum likelihood estimator. . . . . . . . 40

4.7 Uniform generating distributions used to verify the type I error rate for approximate C2STs. Observations falling in the overlapping (light gray) region are indistinguishable, while observations falling in the non-overlapping (dark gray) regions are perfectly distinguishable. . . . . . . . . . . . . . . . . 41

4.8 Empirical type I error and test set classification accuracy for KNN- and NN-based approximate $\mathrm{C} 2 \mathrm{ST}$. . . . . . . . . . . . . . . . . 42

4.9 Empirical power and and test set classification accuracy for KNN- and NN-based approximate $\mathrm{C} 2 \mathrm{ST}$. . . . . . . . . . . . . . . . . . . . 42

4.10 Rejection rates as well as test set classification accuracies for approximate and exact

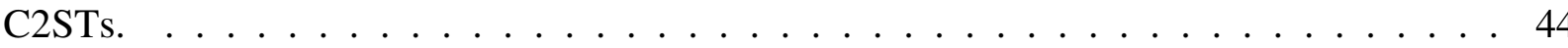

4.11 Permutation importances for the underspecified five-factor fitted model when $N=10000.45$

4.12 C2ST-RFIs for simulated data. The provisory cutoff of 0.9 is marked with a solid line. 46

4.13 Run times for KNN- and NN-based C2STs and C2ST-RFIs from the simulation setting with the underspecified five-factor model. . . . . . . . . . . . . . . . . . 47 


\section{INTRODUCTION}

Item factor analysis (IFA; Bock, Gibbons, \& Muraki 1988) is an invaluable method for investigating the latent structure underlying the discrete item response data that arises in many social science applications. In particular, IFA allows researchers to summarize a large number of item responses using a smaller number of continuous latent factors, thereby reducing the dimensionality of the data and potentially making the data easier to understand. Researchers with specific hypotheses about the number of factors, the relations between the item responses and the factors, and the factor correlations typically encode their hypotheses as parameter constraints in a confirmatory IFA model (e.g., Wirth \& Edwards 2007). For example, personality assessments such as the revised Minnesota Multiphasic Personality Inventory (Butcher, Dahlstrom, Graham, Tellegen, \& Kaemmer 1989) and the International Personality Item Pool NEO (Goldberg 1999) include hundreds of items organized into subscales wherein each subscale is designed to measure a single personality factor. Modeling such designs using confirmatory IFA entails estimating the relations between the items comprising a subscale and their corresponding factor while constraining these items' relations with all other factors to zero (i.e., not estimating these relations). Estimating confirmatory IFA model parameters and their standard errors permits inferences about the properties of items as well as about the characteristics of the population from which the observed sample was drawn. Subsequent goodness-of-fit (GOF) analyses provide useful information about how well the fitted model approximates the data generating model (e.g., Maydeu-Olivares 2013a).

Unfortunately, both parameter estimation and GOF assessment have long been computationally challenging in the large-scale setting with many respondents, items, and latent factors (e.g., Cai 2010a, 2010b). Existing methods for confirmatory IFA may therefore be sub-optimal for analyzing complex, high-dimensional item response data arising from sources such as surveys, 
standardized tests, online applications, and electronic data capture, many of which are becoming increasingly available to social scientists (e.g., Pardos 2017; Woo, Tay, \& Proctor 2020). To clarify this issue, consider Bock and Aitkin's (1981) marginal maximum likelihood (MML) estimator, which has many desirable statistical properties and is typically the preferred estimator for confirmatory IFA parameter estimation. ${ }^{1}$ The MML approach bases inference on the marginal likelihood of the observed item responses, which is obtained by integrating out the latent factors. Problematically, however, evaluating this integral is computationally burdensome when the number of factors $P$ is even moderately large (e.g., $P \geq 5$ ). Researchers have devised numerous methods to avoid this computational burden: adaptive Gaussian quadrature methods (Rabe-Hesketh, Skrondal, \& Pickles 2005; Schilling \& Bock 2005), Laplace approximation methods (e.g., Huber, Ronchetti, \& Victoria-Feser 2004), Monte Carlo expectation-maximization (EM) algorithms (e.g., Meng \& Schilling 1996; Song \& Lee 2005), Markov Chain Monte Carlo methods (e.g., Béguin \& Glas 2001; Edwards 2010), and stochastic approximation methods (SA; e.g., Cai 2010a, 2010b; S. Zhang, Chen, \& Liu 2020). Of the above methods, SA procedures such as the MetropolisHastings Robbins-Monro (MH-RM) algorithm (Cai 2010b) and the stochastic EM (StEM) algorithm (S. Zhang et al. 2020) are the most computationally efficient; in recent years, MH-RM has been particularly widely used in the social and behavioral sciences due to its flexibility and computational efficiency. However, even these state-of-the-art SA procedures are slow when the sample size $N$, number of items $J$, and number of factors $P$ are all large (e.g., $N \geq 10000, J \geq 100$, and $P \geq 10)$.

Even after surmounting the computational difficulties associated with MML estimation, researchers who wish to assess their confirmatory IFA model's GOF face yet another computational barrier. Let $K_{j}$ denote the number of response categories for item $j$. To simplify the presentation, assume all items have the same number of categories such that $K=K_{j}$ for $j=1, \ldots, J$. GOF

\footnotetext{
${ }^{1}$ I note that other estimators such as limited-information estimators (e.g., Jöreskog \& Moustaki 2001; Muthén 1978, 1984) and joint maximum likelihood (JML) estimators (X. Chen, Liu, Sun, \& Hong 2019) are more computationally efficient than the MML estimator. However, these alternative estimators have different statistical properties — for example, limited-information estimators are not asymptotically efficient, while JML estimators are only consistent when the sample size and the number of items simultaneously tend to infinity — and are not considered further here due to space constraints.
} 
assessment for IFA models fitted via MML is typically based on the underlying $K^{J}$-dimensional multinomial table on which the model is defined. Full-information GOF statistics such as Pearson's statistic and the likelihood ratio statistic have inaccurate $p$-values when the number of items and the number of response categories are even moderately large due to the multinomial table's sparseness (e.g., when $J \geq 6$ and $K \geq 5$; Thissen \& Steinberg 1997). Limited-information GOF statistics overcome the sparsity problem by only utilizing marginals of the multinomial table, thereby "concentrating" the information available for testing to obtain more accurate $p$-values and higher power (e.g., Maydeu-Olivares \& Joe 2005, 2006, 2014). However, calculating limited-information GOF statistics requires high-dimensional numerical integration and is typically computationally intensive. Despite efforts to improve computational efficiency for specific IFA models (e.g., Cai \& Hansen 2013), calculating limited-information GOF statistics for general confirmatory IFA models remains computationally intensive when the number of items, response categories, and factors are all large (e.g., $J \geq 100, K \geq 5$, and $P \geq 10$ ).

Based on the preceding discussion, it is clear that more computationally efficient MML estimation and GOF assessment methods are needed to apply confirmatory IFA to very large-scale data. In this thesis, I investigate machine learning methods that offer steps toward addressing some of the difficulties mentioned above. The method I propose for parameter estimation is based on Urban and Bauer's (2021) deep learning algorithm for exploratory IFA. Their algorithm uses an importance-weighted amortized variational estimator (I-WAVE) that combines variational inference and importance sampling to construct an approximation to the MML estimator. By increasing the number of importance-weighted samples drawn during fitting, the I-WAVE typically trades computational efficiency for a better approximation. In the large-scale exploratory setting (i.e., $P=10, J=100,1000 \leq N \leq 10000$ ), the I-WAVE has empirically demonstrated comparable parameter estimation accuracy and increased computational efficiency relative to the MML estimator implemented via MH-RM (Urban \& Bauer 2021).

Assessing GOF for large-scale IFA models fitted via I-WAVE is not straightforward. In addition 
to being computationally inefficient in the large-scale setting, the limited-information GOF statistics described above were designed for models fitted via the MML estimator and have unknown theoretical properties when applied to models fitted via approximate MML (e.g., the I-WAVE). I aim to address both computational and theoretical issues simultaneously by instead considering simulation-based GOF assessment methods in which model-data fit is assessed by comparing the observed item responses to synthetic item responses sampled from a fitted IFA model. Previous work in simulation-based GOF assessment for confirmatory IFA has mainly focused on posterior predictive model checking (PPMC) in the Bayesian setting (e.g., Levy, Mislevy, \& Sinharay 2009; Sinharay, Johnson, \& Stern 2006). In PPMC, synthetic data simulated from the posterior predictive distribution are compared to the observed data using discrepancy measures (i.e., measures that quantify how two data sets differ). Unlike limited-information GOF statistics, PPMC is computationally efficient, accounts for parameter estimation uncertainty, and does not rely on asymptotic arguments. Although PPMC was originally developed for Bayesian IFA models, recent work has shown that PPMC may be applied to frequentist IFA models using a normal approximation to the posterior predictive distribution (Kuhfeld 2019; Lee, Cai, \& Kuhfeld 2016). Unfortunately, PPMC assuming posterior normality (PPMC-N) is only well-motivated when models are fitted via exact maximum likelihood, suggesting that PPMC-N may not be a well-motivated GOF assessment method for I-WAVE.

The alternative GOF assessment methods I consider are based on a class of machine learning methods called classifier two-sample tests (C2STs; Friedman 2003; Kim, Ramdas, Singh, \& Wasserman 2021; Lopez-Paz \& Oquab 2017). Similar to $t$-tests and other classical two-sample tests, C2STs aim to determine whether two samples are drawn from the same distribution. To assess model-data fit in confirmatory IFA, a C2ST begins by first sampling synthetic item responses from the fitted IFA model. ${ }^{2}$ The synthetic responses are combined with the observed responses to construct a new data set, which is divided at random into two disjoint subsets called the training

\footnotetext{
${ }^{2}$ Unlike PPMC-N, the C2ST is well-motivated when applied to an IFA model fitted via any estimator given that synthetic data can be sampled from the model. This holds for estimators that treat the latent factors as random effects (e.g., the MML estimator or the I-WAVE) but not for estimators that treat the latent factors as fixed effects (e.g., JML estimators).
} 
set and the test set. Next, a machine learning classifier (e.g., a logistic regression or a neural network classifier) is trained to distinguish between the observed and synthetic training set response patterns. Finally, the classifier's test set accuracy (i.e., the proportion of test set response patterns correctly classified as observed or synthetic) is treated as the test statistic and is used to test the null hypothesis that the observed and synthetic data are drawn from the same distribution. For a perfect-fitting model, the accuracy obtained should not be significantly better than chance because the synthetic data will mimic the observed data characteristics. Depending on one's choice for the classifier, C2STs are potentially very computationally efficient in the large-scale setting. C2STs also provide a variety of interpretable values to complement the use of $p$-values and may be viewed as uniting overall model fit, piece-wise (i.e., item-level) fit, and person fit under a single framework.

In exploring these issues, my thesis makes four primary contributions. First, I extend the IWAVE to the confirmatory setting by showing how to handle user-defined constraints on the factor loadings and inter-factor correlations. Second, I empirically investigate confirmatory I-WAVE's finite sample behavior and conduct comparisons with MH-RM. Third, in addition to a C2ST-based test of perfect (exact) fit, I propose and explore a novel C2ST-based test of approximate fit as well as a C2ST-based relative fit index that is similar in spirit to the relative fit indices used in linear confirmatory factor analysis and structural equation modeling (SEM; e.g., Bentler 1990; Bentler \& Bonett 1980; Bollen 1989b; L. R. Tucker \& Lewis 1973). Compared to standard C2STs, these new methods are potentially better suited to applications where the specified IFA model is unlikely to exactly capture the data generating model. Fourth, I conduct simulations to investigate the C2ST variants' finite sample behavior in several settings including when the IFA model is correctly and incorrectly specified.

The remaining chapters are organized as follows. Chapter 2 reviews variational methods (e.g., I-WAVE) for fitting confirmatory IFA models with polytomous responses. Novel C2ST-based GOF assessment methods are developed in Chapter 3. Chapter 4 includes implementation details for the proposed parameter estimation and GOF assessment methods as well as an empirical example and 
simulation studies. Experimental results are summarized, then limitations and extensions of the proposed methods are discussed in Chapter 5. 


\section{VARIATIONAL METHODS FOR PARAMETER ESTIMATION}

Variational inference (VI) is an approach to approximate maximum likelihood estimation for latent variable (LV) models that is widely used in machine learning (Blei, Kucukelbir, \& McAuliffe 2017; C. Zhang, Butepage, Kjellstrom, \& Mandt 2019). VI has recently been applied for IFA in a variety of settings (e.g., Y. Chen, Filho, Prudêncio, Diethe, \& Flach 2019; Cho, Wang, Zhang, \& Xu 2020; Curi, Converse, \& Hajewski 2019; Hui, Warton, Ormerod, Haapaniemi, \& Taskinen 2017; Natesan, Nandakumar, Minka, \& Rubright 2016; Urban \& Bauer 2021; Wu, Davis, Domingue, Piech, \& Goodman 2020). In this chapter, I review variational methods for IFA. I focus in particular on the VI-based method described by Urban and Bauer (2021), which provides a theoretical link between VI and MML estimation.

\subsection{A Model for Confirmatory IFA}

I first establish notation for the IFA models under consideration. Specifically, I consider Samejima's (1969) graded response model (GRM) for polytomous item responses, although the methods discussed in this chapter readily apply to other IFA models. Suppose that $N$ respondents have answered $J$ items. Let $x_{i, j} \in\left\{0,1, \ldots, K_{j}-1\right\}$ denote the response for respondent $i$ to item $j$ in $K_{j}$ ordinal categories. To simplify the presentation, assume $K_{j}=K$ for $j=1, \ldots, J$. Note that when $K=2$, the GRM reduces to the widely used multidimensional two-parameter logistic model (McKinley \& Reckase 1983).

Each respondent is represented by a $P \times 1$ latent vector $\mathbf{z}_{i}$ and each item is represented by a $(P+K-1) \times 1$ parameter vector $\boldsymbol{\theta}_{j}=\left(\boldsymbol{\alpha}_{j}^{\top}, \boldsymbol{\beta}_{j}^{\top}\right)^{\top}$ where $\boldsymbol{\beta}_{j}$ is a $P \times 1$ vector of loadings and $\boldsymbol{\alpha}_{j}=\left(\alpha_{j 1}, \ldots, \alpha_{j, K-1}\right)^{\top}$ is a $(K-1) \times 1$ vector of strictly ordered category intercepts. The GRM defines a set of boundary response probabilities conditional on $\boldsymbol{\theta}_{j}$ and $\mathbf{z}_{i}$ :

$$
\operatorname{Pr}\left(x_{i, j} \geq k \mid \boldsymbol{\theta}_{j}, \mathbf{z}_{i}\right)=\frac{1}{1+\exp \left[\alpha_{j, k}+\boldsymbol{\beta}_{j}^{\top} \mathbf{z}_{i}\right]}, \quad k \in\{1, \ldots, K-1\},
$$


where $\operatorname{Pr}\left(x_{i, j} \geq 0 \mid \boldsymbol{\theta}_{j}, \mathbf{z}_{i}\right)=1$ and $\operatorname{Pr}\left(x_{i, j} \geq K \mid \boldsymbol{\theta}_{j}, \mathbf{z}_{i}\right)=0$. The conditional probability of the response $x_{i, j}=k$ for $k \in\{0, \ldots K-1\}$ is

$$
\pi_{i, j, k}=\operatorname{Pr}\left(x_{i, j}=k \mid \boldsymbol{\theta}_{j}, \mathbf{z}_{i}\right)=\operatorname{Pr}\left(x_{i, j} \geq k \mid \boldsymbol{\theta}_{j}, \mathbf{z}_{i}\right)-\operatorname{Pr}\left(x_{i, j} \geq k+1 \mid \boldsymbol{\theta}_{j}, \mathbf{z}_{i}\right)
$$

It follows from Equation (2.2) that the conditional distribution of $x_{i, j}$ is multinomial with $K$ cells, trial size 1 , and cell probabilities $\pi_{i, j, k}$ :

$$
p_{\boldsymbol{\theta}_{j}}\left(x_{i, j} \mid \mathbf{z}_{i}\right)=\prod_{k=0}^{K-1} \pi_{i, j, k}^{\mathbb{1}\left(x_{i, j}=k\right)}
$$

where $\mathbb{1}(\cdot)$ denotes the indicator function. Let $\mathbf{x}_{i}=\left(x_{i, 1}, \ldots, x_{i, n}\right)^{\top}$ be the $i^{\text {th }}$ respondent's response pattern and let $\boldsymbol{\theta}=\left(\boldsymbol{\theta}_{1}^{\top}, \ldots, \boldsymbol{\theta}_{J}^{\top}\right)^{\top}$ be a vector collecting all item parameters. By the local independence assumption, the conditional distribution of $\mathbf{x}_{i}$ is

$$
p_{\boldsymbol{\theta}}\left(\mathbf{x}_{i} \mid \mathbf{z}_{i}\right)=\prod_{j=1}^{J} p_{\boldsymbol{\theta}_{j}}\left(x_{i, j} \mid \mathbf{z}_{i}\right)
$$

Assume that $\mathbf{z}_{i}$ is multivariate normally distributed with zero mean vector and covariance matrix $\boldsymbol{\Sigma}=\left(\boldsymbol{\sigma}_{p, p^{\prime}}\right)_{P \times P}$. Let $\boldsymbol{\omega}=\left(\boldsymbol{\theta}^{\top}, \operatorname{vech}(\boldsymbol{\Sigma})^{\top}\right)^{\top}$ be a vector collecting all unknown parameters where $\operatorname{vech}(\boldsymbol{\Sigma})$ stacks the $P(P+1) / 2$ unique elements of $\Sigma$ into a vector. Under my assumptions about the distribution of the factors, the marginal distribution of $\mathbf{x}_{i}$ is given by

$$
p_{\boldsymbol{\omega}}\left(\mathbf{x}_{i}\right)=\int \prod_{j=1}^{J} p_{\boldsymbol{\theta}_{j}}\left(x_{i, j} \mid \mathbf{z}\right) \mathscr{N}(\mathbf{z} \mid \mathbf{\Sigma}) d \mathbf{z}
$$

where $\mathscr{N}(\cdot \mid \boldsymbol{\Sigma})$ is a normal density parameterized by $\boldsymbol{\Sigma}$ and the above integral is over $\mathbb{R}^{P}$.

I set $\sigma_{p, p}=1$ for $p=1, \ldots, P$ to identify the scale of the factors. In the confirmatory setting, users encode hypotheses about the measurement structure by placing restrictions on the loadings (e.g., by fixing $\beta_{j, p}$ to zero if item $j$ is not hypothesized to measure factor $p$ ). Following Cai 
(2010b), I consider the case of linear equality constraints so that the loadings may be written as

$$
\boldsymbol{\beta}_{j}=\mathbf{b}_{j}+\mathbf{A}_{j} \boldsymbol{\beta}_{j}^{\prime}
$$

where $\boldsymbol{\beta}_{j}$ is the restricted loadings vector, $\mathbf{b}_{j}$ is a $P \times 1$ vector of constants, $\mathbf{A}_{j}$ is a $P \times P$ matrix of constants that implements the linear constraints, and $\boldsymbol{\beta}_{j}^{\prime}$ is a vector of free parameters. Cai (2010b) provides examples of how $\mathbf{b}_{j}$ and $\mathbf{A}_{j}$ may be specified to implement various restrictions, while Anderson and Rubin (1956) provide sufficient conditions enabling $\mathbf{b}_{j}$ and $\mathbf{A}_{j}$ to be specified such that the model is identified.

\subsection{Variational Lower Bound}

Let $\mathbf{X}$ be an $N \times J$ matrix whose $i^{\text {th }}$ row is $\mathbf{x}_{i}^{\top}$. The marginal log-likelihood of the observed data is

$$
\left.\ell(\boldsymbol{\omega} \mid \mathbf{X})=\sum_{i=1}^{N} \log \left[\int \prod_{j=1}^{J} p_{\boldsymbol{\theta}_{j}}\left(x_{i, j} \mid \mathbf{z}_{i}\right) \mathscr{N}(\mathbf{z} \mid \mathbf{\Sigma}) d \mathbf{z}\right)\right] .
$$

Maximizing $\ell(\boldsymbol{\omega} \mid \mathbf{X})$ by directly evaluating the $N$ integrals in Equation (2.7) is computationally intensive when $P$ is large. VI solves this issue by instead maximizing a computationally tractable lower bound on $\ell(\boldsymbol{\omega} \mid \mathbf{X})$. To derive the lower bound, I first re-write a single summand in Equation (2.7) as

$$
\log p_{\boldsymbol{\omega}}\left(\mathbf{x}_{i}\right)=D_{\mathrm{KL}}\left[q \boldsymbol{\Psi}_{i}\left(\mathbf{z}_{i}\right) \| p_{\boldsymbol{\omega}}\left(\mathbf{z}_{i} \mid \mathbf{x}_{i}\right)\right]+\mathbb{E}_{q_{\boldsymbol{\psi}_{i}}\left(\mathbf{z}_{i}\right)}\left[\log p_{\boldsymbol{\omega}}\left(\mathbf{z}_{i}, \mathbf{x}_{i}\right)-\log q_{\boldsymbol{\Psi}_{i}}\left(\mathbf{z}_{i}\right)\right]
$$

where $D_{\mathrm{KL}}[\cdot \| \cdot]$ denotes the Kullback-Leibler $(\mathrm{KL})$ divergence ${ }^{1}$ and $q_{\boldsymbol{\psi}_{i}}\left(\mathbf{z}_{i}\right)$ is an arbitrary density with parameter vector $\boldsymbol{\psi}_{i}$ satisfying $\int q \boldsymbol{\Psi}_{i}(\mathbf{z}) d \mathbf{z}=1$. The first r.h.s. term in Equation (2.8) is the KL divergence between $q \boldsymbol{\psi}_{i}\left(\mathbf{z}_{i}\right)$ and the posterior distribution of the latent factors. Since this term in nonnegative, the second r.h.s. term is a lower bound on the marginal log-likelihood of a single observation:

$$
\log p_{\boldsymbol{\omega}}\left(\mathbf{x}_{i}\right) \geq \mathbb{E}_{q_{\boldsymbol{\psi}_{i}\left(\mathbf{z}_{i}\right)}}\left[\log p_{\boldsymbol{\omega}}\left(\mathbf{z}_{i}, \mathbf{x}_{i}\right)-\log q_{\boldsymbol{\Psi}_{i}}\left(\mathbf{z}_{i}\right)\right]
$$

\footnotetext{
${ }^{1}$ The $\mathrm{KL}$ divergence is defined as $D_{\mathrm{KL}}[\mathbb{Q} \| \mathbb{P}]=\mathbb{E}_{\mathbb{Q}}[\log \mathbb{Q}]-\mathbb{E}_{\mathbb{Q}}[\log \mathbb{P}]$ for distributions $\mathbb{P}$ and $\mathbb{Q}$. It satisfies $D_{\mathrm{KL}}[\mathbb{Q} \| \mathbb{P}] \geq 0$ with equality if and only if $\mathbb{P}=\mathbb{Q}$ almost everywhere.
} 
This lower bound is called the evidence lower bound (ELBO) and is often re-written as:

$$
\begin{aligned}
\mathrm{ELBO}_{i} & =\mathbb{E}_{q_{\boldsymbol{\psi}_{i}}\left(\mathbf{z}_{i}\right)}\left[\log p_{\boldsymbol{\omega}}\left(\mathbf{z}_{i}, \mathbf{x}_{i}\right)-\log q_{\boldsymbol{\psi}_{i}}\left(\mathbf{z}_{i}\right)\right] \\
& =\mathbb{E}_{q_{\boldsymbol{\psi}_{i}}\left(\mathbf{z}_{i}\right)}\left[\log p_{\boldsymbol{\omega}}\left(\mathbf{x}_{i} \mid \mathbf{z}_{i}\right)\right]-D_{\mathrm{KL}}\left[q_{\boldsymbol{\psi}_{i}}\left(\mathbf{z}_{i}\right) \| \mathscr{N}\left(\mathbf{z}_{i} \mid \boldsymbol{\Sigma}\right)\right]
\end{aligned}
$$

The variational estimator of the IFA model parameters $\boldsymbol{\omega}$ is obtained by maximizing the ELBO over all observations w.r.t. both $\boldsymbol{\omega}$ and $\boldsymbol{\psi}_{i}$, which is equivalent to minimizing the KL divergence between $q \boldsymbol{\Psi}_{i}\left(\mathbf{z}_{i}\right)$ and the posterior distribution of the latent factors. Intuitively, obtaining the variational estimator drives $q_{\boldsymbol{\Psi}_{i}}\left(\mathbf{z}_{i}\right)$ to approximate the true LV posterior; I henceforth refer to $q_{\boldsymbol{\psi}_{i}}\left(\mathbf{z}_{i}\right)$ as the approximate LV posterior. When the approximate LV posterior perfectly approximates the true posterior such that the KL divergence term on the r.h.s. of Equation (2.8) is zero, the ELBO equals the marginal log-likelihood and the variational estimator is equivalent to the MML estimator.

Following previous work (e.g., Hui et al. 2017; Kingma \& Welling 2014; Urban \& Bauer 2021), I set the approximate LV posterior to the isotropic normal density:

$$
q \boldsymbol{\Psi}_{i}\left(\mathbf{z}_{i}\right)=\mathscr{N}\left(\mathbf{z}_{i} \mid \boldsymbol{\mu}_{i}, \boldsymbol{\sigma}_{i}^{2} \mathbf{I}_{P}\right)
$$

where $\boldsymbol{\mu}_{i}$ is a $P \times 1$ vector of means, $\boldsymbol{\sigma}_{i}^{2}$ is a $P \times 1$ vector of variances, and $\mathbf{I}_{P}$ is a $P \times P$ identity matrix. Although the isotropic normal approximate LV posterior is computationally tractable, it is rarely flexible enough in practice to perfectly approximate the true posterior and thereby minimize the KL divergence to zero. The importance sampling technique described in Sect. 2.4 decreases the gap between the ELBO and the marginal log-likelihood by implicitly increasing the flexibility of the approximate posterior.

\subsection{Amortized VI}

Traditional VI fits a different parameter vector $\boldsymbol{\psi}_{i}$ (i.e., a different approximate LV posterior) for each observation. This approach quickly becomes computationally infeasible for large sample sizes. Additionally, it is not immediately clear how to apply models fitted using VI to new observations (e.g., to compute the log-likelihood of the new observations). Amortized variational 
inference (AVI) solves the above issues by parameterizing the approximate posterior using a powerful function approximator called an inference model. Since the inference model parameters are shared across observations, performing AVI requires fitting a constant number of parameters regardless of the sample size, whereas performing VI requires fitting a number of parameters that grows linearly with the sample size. Models fitted using AVI can also be straightforwardly applied to new observations by passing the observations through the inference model.

The variational autoencoder (VAE; Kingma \& Welling 2014; Rezende, Mohamed, \& Wierstra 2014) is an algorithm for AVI that uses an artificial neural network (NN) inference model. NNs are parametric machine learning models that map a set of predictor variables through a sequence of transformations to predict a set of outcome variables (for a brief overview, see Urban \& Bauer 2021). Using an NN inference model is typically considered justifiable because NNs can approximate any Borel measurable function (e.g., Cybenko 1989) and perform well in a variety of real-world applications (LeCun, Bengio, \& Hinton 2015). I can specify a VAE for confirmatory IFA by parameterizing the approximate LV posterior as follows:

$$
\begin{aligned}
\left(\boldsymbol{\mu}_{i}^{\top}, \log \boldsymbol{\sigma}_{i}^{\top}\right)^{\top} & =\mathrm{NN}_{\boldsymbol{\psi}}\left(\mathbf{x}_{i}\right), \\
q_{\boldsymbol{\psi}}\left(\mathbf{z}_{i} \mid \mathbf{x}_{i}\right) & =\mathscr{N}\left(\mathbf{z}_{i} \mid \boldsymbol{\mu}_{i}, \boldsymbol{\sigma}_{i}^{2} \mathbf{I}_{P}\right),
\end{aligned}
$$

where $\boldsymbol{\mu}_{i}$ is a $P \times 1$ predicted vector of means, $\log \boldsymbol{\sigma}_{i}$ is a $P \times 1$ predicted vector of $\log$-standard deviations, and $\mathrm{NN}_{\boldsymbol{\psi}}$ is a neural network parameterized by $\boldsymbol{\psi}$. Instead of estimating a parameter vector $\boldsymbol{\psi}_{i}$ for each observation, the NN parameters $\boldsymbol{\psi}$ are now shared across observations. That is, instead of maximizing Equation (2.11) over observations, I maximize

$$
\mathrm{ELBO}=\mathbb{E}_{q_{\boldsymbol{\psi}}(\mathbf{z} \mid \mathbf{x})}\left[\log p_{\boldsymbol{\omega}}(\mathbf{x} \mid \mathbf{z})\right]-D_{\mathrm{KL}}\left[q_{\boldsymbol{\psi}}(\mathbf{z} \mid \mathbf{x}) \| \mathscr{N}(\mathbf{z} \mid \boldsymbol{\Sigma})\right]
$$

over observations. Note that I have dropped the case index $i$ since $\boldsymbol{\psi}$ is shared across $\left\{\mathbf{x}_{i}, \mathbf{z}_{i}\right\}_{i=1}^{N}$.

The VAE relies on the assumption that the $\mathrm{NN}$ is capable of approximating the mapping from the data space to the approximate LV posterior parameter space. In theory, this assumption holds 
when the NN is sufficiently flexible (e.g., when the NN has one infinitely large hidden layer). In practice, however, the NN has a limited capacity that may prevent the VAE from performing as well as traditional VI. Fortunately, this performance difference can be reduced by increasing the flexibility of the approximate LV posterior (Cremer, Li, \& Duvenaud 2018).

Technical details regarding model fitting are briefly reviewed here; see Urban and Bauer (2021) for greater detail. The fitting procedure of Urban and Bauer (2021) requires an unbiased estimator for the gradient of the ELBO w.r.t. $\boldsymbol{\xi}=\left(\boldsymbol{\omega}^{\top}, \boldsymbol{\psi}^{\top}\right)^{\top}$. This is obtained by first reparameterizing $\mathbf{z}$ as

$$
\begin{aligned}
& \boldsymbol{\epsilon} \sim \mathscr{N}(\boldsymbol{\epsilon}), \\
& \mathbf{z}=\boldsymbol{\mu}+\boldsymbol{\sigma} \odot \boldsymbol{\epsilon},
\end{aligned}
$$

where $\boldsymbol{\epsilon}$ is a $P \times 1$ sample from a standard multivariate normal density, $\boldsymbol{\mu}$ and $\boldsymbol{\sigma}$ are the outputs of the NN inference model given in equations 2.13 , and $\odot$ denotes element-wise multiplication. This reparameterization writes $\mathbf{z}$ as a deterministic function of $\boldsymbol{\psi}$ and can be viewed as "externalizing" the randomness in z. An unbiased estimator for the gradient of the ELBO w.r.t. $\boldsymbol{\xi}$ is now given by

$$
\begin{aligned}
\nabla_{\boldsymbol{\xi}} \mathrm{ELBO} & =\mathbb{E}_{\mathscr{N}(\boldsymbol{\epsilon})}\left[\nabla_{\boldsymbol{\xi}} \log p_{\boldsymbol{\omega}}(\mathbf{z}, \mathbf{x})-\nabla_{\boldsymbol{\xi}} \log q_{\boldsymbol{\psi}}(\mathbf{z} \mid \mathbf{x})\right] \\
& \approx \frac{1}{S} \sum_{s=1}^{S}\left[\nabla_{\boldsymbol{\xi}} \log p_{\boldsymbol{\omega}}\left(\mathbf{z}_{s}, \mathbf{x}\right)-\nabla_{\boldsymbol{\xi}} \log q_{\boldsymbol{\psi}}\left(\mathbf{z}_{S} \mid \mathbf{x}\right)\right]
\end{aligned}
$$

where $\nabla_{\xi}$ returns an $M \times 1$ vector of first-order partial derivatives w.r.t. $\boldsymbol{\xi}$ and (2.17) is a Monte Carlo approximation to the expectation in (2.16). Figure 2.1 illustrates how computation proceeds in a VAE for confirmatory IFA: First, an item response $\mathbf{x}$ is passed through the inference model $\mathrm{NN}_{\boldsymbol{\psi}}$ to produce the approximate $\mathrm{LV}$ posterior parameters $\left(\boldsymbol{\mu}^{\top}, \log \boldsymbol{\sigma}^{\top}\right)^{\top}$; next, a latent vector $\mathbf{z}$ is sampled from $\mathscr{N}\left(\mathbf{z} \mid \boldsymbol{\mu}, \boldsymbol{\sigma}^{2} \mathbf{I}_{P}\right)$ and used to compute the response probabilities $\pi_{j, k}$ for $j=1, \ldots, 6$ and $k=0, \ldots, 2$; finally, these intermediate quantities are used to compute both the expected conditional log-likelihood and the KL divergence terms in the ELBO. The gradient estimator in Equation (2.17) can be efficiently computed using an automatic differentiation procedure called backpropagation (e.g., Goodfellow, Bengio, \& Courville 2016). Urban and Bauer (2021) then apply an 


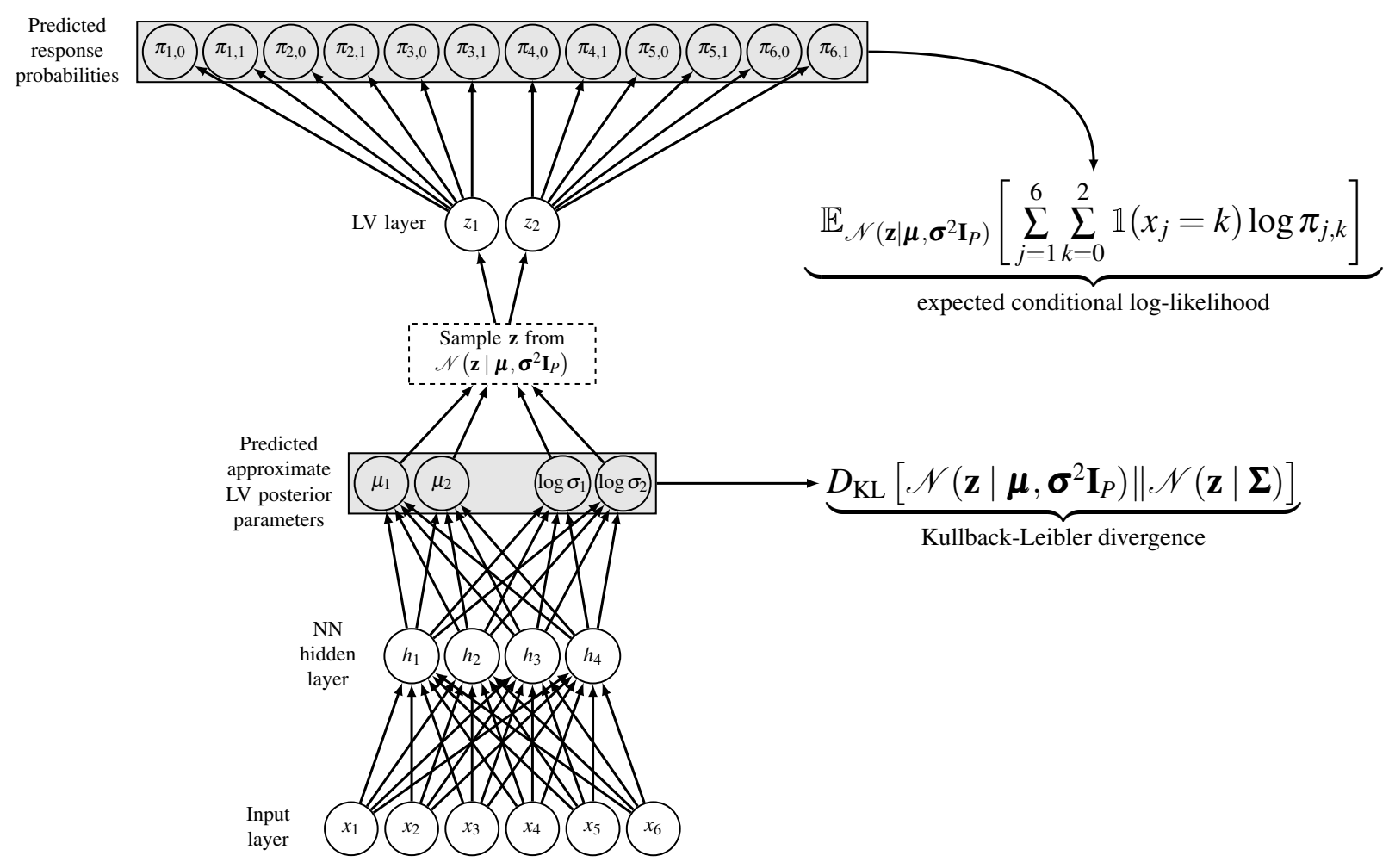

Figure 2.1: Schematic diagram of a variational autoencoder for confirmatory item factor analysis with $J=6$ items, $K=2$ categories per item, $P=2$ factors, $S=1$ Monte Carlo sample from the approximate latent variable posterior, and an inference model consisting of a neural network with a single hidden layer. Each factor loads on three items. The reparameterization of $\mathbf{z}$ is not illustrated for simplicity. $\mathrm{LV}=$ latent variable, $\mathrm{NN}=$ neural network.

adaptive stochastic gradient method called AMSGrad (Reddi, Kale, \& Kumar 2018) to iteratively update $\xi$ until convergence.

\subsection{Importance-Weighted VI}

Importance-weighted VI (Burda, Grosse, \& Salakhutdinov 2016; Domke \& Sheldon 2018) is a strategy for obtaining a better approximation to the true log-likelihood by increasing the flexibility of traditional VI. The importance-weighted amortized variational estimator (I-WAVE) for the IFA model parameters $\boldsymbol{\omega}$ is obtained by maximizing a new lower bound called the importance-weighted 
ELBO (IW-ELBO):

$$
\begin{aligned}
\log p_{\boldsymbol{\omega}}(\mathbf{x}) & \geq \text { IW-ELBO } \\
& =\mathbb{E}_{\mathbf{z}_{1: R}}\left[\log \frac{1}{R} \sum_{r=1}^{R} w_{r}\right]
\end{aligned}
$$

where $\mathbf{z}_{1: R} \sim \prod_{r=1}^{R} q_{\boldsymbol{\psi}}\left(\mathbf{z}_{r} \mid \mathbf{x}\right), w_{r}=p_{\boldsymbol{\omega}}\left(\mathbf{z}_{r}, \mathbf{x}\right) / q_{\boldsymbol{\psi}}\left(\mathbf{z}_{r} \mid \mathbf{x}\right)$ are unnormalized importance weights for the joint distribution of latent and observed variables, and $R$ is the number of importance-weighted (IW) samples. The IW-ELBO reduces to the ELBO when $R=1$ and converges monotonically to the marginal log-likelihood as $R \rightarrow \infty$ under mild assumptions (Burda et al. 2016). I-WAVE can also be viewed as implicitly defining a flexible approximate LV posterior $q_{\boldsymbol{\psi}}^{\mathrm{IW}}(\mathbf{z} \mid \mathbf{x})$ that approaches the true LV posterior pointwise as $R \rightarrow \infty$ (Cremer et al., 2017). The above facts imply that I-WAVE and the MML estimator are equivalent when the number of IW samples $R$ equals infinity, in which case I-WAVE inherits the MML estimator's statistical properties.

Following Urban and Bauer (2021), I use Burda et al.'s (2016) estimator for the IW-ELBO $\boldsymbol{\omega}$-gradient:

$$
\begin{aligned}
\nabla_{\boldsymbol{\omega}} \mathbb{E}_{\mathbf{x}_{1: R}}\left[\log \frac{1}{R} \sum_{r=1}^{R} w_{r}\right] & =\mathbb{E}_{\boldsymbol{\epsilon}_{1: R}}\left[\sum_{r=1}^{R} \widetilde{w}_{r} \nabla_{\boldsymbol{\omega}} \log w_{r}\right] \\
& \approx \frac{1}{S} \sum_{s=1}^{S}\left[\sum_{r=1}^{R} \widetilde{w}_{r, s} \nabla_{\boldsymbol{\omega}} \log w_{r, s}\right]
\end{aligned}
$$

as well as G. Tucker, Maddison, Lawson, and Gu's (2019) “doubly reparameterized” estimator for the IW-ELBO $\psi$-gradient:

$$
\begin{aligned}
\nabla_{\boldsymbol{\psi}} \mathbb{E}_{\mathbf{x}_{1: R}}\left[\log \frac{1}{R} \sum_{r=1}^{R} w_{r}\right] & =\mathbb{E}_{\boldsymbol{\epsilon}_{1: R}}\left[\sum_{r=1}^{R} \widetilde{w}_{r}^{2} \frac{\partial \log w_{r}}{\partial \mathbf{z}_{r}} \frac{\partial \mathbf{z}_{r}}{\partial \boldsymbol{\psi}}\right]^{\top} \\
& \approx \frac{1}{S} \sum_{s=1}^{S}\left[\sum_{r=1}^{R} \widetilde{w}_{r, s}^{2} \frac{\partial \log w_{r, s}}{\partial \mathbf{z}_{r, s}} \frac{\partial \mathbf{z}_{r, s}}{\partial \boldsymbol{\psi}}\right]^{\top},
\end{aligned}
$$

where $\boldsymbol{\epsilon}_{1: R} \sim \prod_{r=1}^{R} \mathscr{N}\left(\boldsymbol{\epsilon}_{r}\right)$ and $\widetilde{w}_{r}=w_{r} / \sum_{r^{\prime}=1}^{R} w_{r^{\prime}}$ are normalized importance weights. Both estimators are unbiased and have increasing signal-to-noise ratios as $R \rightarrow \infty$. Further, both can be 
successfully approximated using a single Monte Carlo sample (e.g., Burda et al. 2016; G. Tucker et al. 2019), so I set $S=1$ for all experiments in this thesis.

\subsection{Handling User-Defined Constraints}

User-defined constraints on the factor loadings are straightforward to implement for I-WAVE. In particular, the gradient of the IW-ELBO w.r.t. the unconstrained loadings vector $\boldsymbol{\beta}_{j}^{\prime}$ can be obtained using the chain rule:

$$
\nabla_{\boldsymbol{\beta}_{j}^{\prime}} \mathrm{IW}-\mathrm{ELBO}=\left(\frac{\partial \mathrm{IW}-\mathrm{ELBO}}{\partial \boldsymbol{\beta}_{j}} \frac{\partial \boldsymbol{\beta}_{j}}{\partial \boldsymbol{\beta}_{j}^{\prime}}\right)^{\top}=\mathbf{A}_{j}^{\top} \nabla_{\boldsymbol{\beta}_{j}} \text { IW-ELBO }
$$

Equation (2.24) implies that one can first compute the gradient of the IW-ELBO w.r.t. $\boldsymbol{\beta}_{j}$, then obtain the gradient w.r.t. $\boldsymbol{\beta}_{j}^{\prime}$ via pre-multiplication by the transposed constraint matrix $\mathbf{A}_{j}^{\top}$.

Users also often wish to impose constraints on the factor correlation matrix $\boldsymbol{\Sigma}$. Let $\boldsymbol{\Sigma}=\mathbf{L L}^{\top}$ where $\mathbf{L}$ is a $P \times P$ lower triangular matrix. I estimate $\mathbf{L}$ using a hyperspherical parameterization (Pinheiro \& Bates 1996; Rapisarda, Brigo, \& Mercurio 2007), which enables unconstrained estimation of a variety of structured correlation matrices and has similar computational efficiency to estimating $\mathbf{L}$ directly. This parameterization is given by:

$$
l_{p, p^{\prime}}= \begin{cases}\cos \vartheta_{p, 1}, & \text { if } p^{\prime}=1 \\ \cos \vartheta_{p, p^{\prime}+1} \prod_{p^{\prime \prime}=1}^{p^{\prime}} \sin \vartheta_{p, p^{\prime \prime}}, & \text { if } 1<p^{\prime}<p \\ \prod_{p^{\prime \prime}=1}^{p} \sin \vartheta_{p, p^{\prime \prime}}, & \text { if } p^{\prime}=p,\end{cases}
$$

for $p=1, \ldots, P$ where $l_{p, p^{\prime}}$ are elements of $\mathbf{L}$ and $\vartheta_{p, p^{\prime}} \in(0, \pi]$ are angles measured in radians which are elements of a $P \times P$ lower triangular matrix $\Theta$. Constraints on the angles giving rise to various correlation structures are discussed by Tsay and Pourahmadi (2017) as well as by Ghosh, Mallick, and Pourahmadi (2020). I note that it is also feasible to estimate $\boldsymbol{\Sigma}$ using a proximal stochastic gradient method (e.g., Yun, Lozano, \& Yang 2020). 


\subsection{Summary}

In this chapter, I introduced notation for a class of confirmatory IFA models for polytomous items response data and derived a computationally tractable lower bound on the marginal loglikelihood of the observed data. I showed how to combine the lower bound with both deep learning and importance sampling to develop an importance-weighted amortized variational estimator for the IFA model parameters that is equivalent to the MML estimator when the number of importance-weighted samples equals infinity. I also discussed how to parameterize the IFA model to incorporate user-defined constraints on the factor loadings and the factor correlation matrix. These developments will be investigated experimentally in a later chapter. 


\section{CLASSIFIER TWO-SAMPLE TESTS FOR GOODNESS-OF-FIT ASSESSMENT}

\subsection{Exact C2STs}

I now discuss the application of classifier two-sample tests (C2STs; Friedman 2003; Kim et al. 2021; Lopez-Paz \& Oquab 2017), a class of simulation-based GOF assessment methods that have recently been developed in machine learning, to assessing exact GOF for confirmatory IFA models. Let $\hat{\boldsymbol{\omega}}$ denote parameter estimates obtained for some confirmatory IFA model. Let $\mathbf{x}_{i} \sim \mathbb{P}$ denote the $i^{\text {th }}$ observed response pattern and let $\mathbf{y}_{j} \sim p_{\hat{\boldsymbol{\omega}}}\left(\mathbf{y}_{j}\right)=\hat{\mathbb{P}}$ denote the the $j^{\text {th }}$ synthetic response pattern drawn from the fitted model where $\mathbf{x}_{i}, \mathbf{y}_{j} \in \mathscr{X}$ for $i=1, \ldots, N_{1}$ and $j=1, \ldots, N_{2}$. To simplify the presentation, I assume $N_{1}=N_{2}=N$.

C2STs aim to test whether the observed and synthetic response patterns are drawn from the same distribution - that is, C2STs aim to test $H_{0}: \mathbb{P}=\hat{\mathbb{P}}$ against $H_{1}: \mathbb{P} \neq \hat{\mathbb{P}}$. A C2ST is conducted by training a machine learning classifier to distinguish between the observed and synthetic response

patterns. Intuitively, when $\mathbb{P}=\hat{\mathbb{P}}$, the classifier's test set accuracy should be close to $1 / 2$ (i.e., chance), since samples from $\mathbb{P}$ and $\hat{\mathbb{P}}$ are indistinguishable. When $\mathbb{P} \neq \hat{\mathbb{P}}$, the classifier should be able to capitalize on the distributional differences to obtain a test set accuracy higher than $1 / 2$. More formally, a C2ST can be described as the following five-step procedure (Lopez-Paz \& Oquab 2017):

(1) Construct a data set $D=\left\{\left(\mathbf{x}_{i}, 1\right)\right\}_{i=1}^{N} \cup\left\{\left(\mathbf{y}_{i}, 0\right)\right\}_{i=1}^{N}=\left\{\left(\mathbf{u}_{i}, l_{i}\right)\right\}_{i=1}^{2 N}$.

(2) Shuffle $D$ at random and split it into disjoint sets $D=D_{\text {train }} \cup D_{\text {test }}$ where $N_{\text {train }}=\left|D_{\text {train }}\right|$ and $N_{\text {test }}=\left|D_{\text {test }}\right|$.

(3) Fit a machine learning classifier $\hat{f}: \mathscr{X} \rightarrow[0,1]$ using $D_{\text {train }}$. 
(4) Obtain the test set classification accuracy:

$$
\widehat{a c c}=\frac{1}{N_{\text {test }}} \sum_{i \in I_{\text {test }}} \mathbb{1}\left(\mathbb{1}\left(\hat{f}\left(\mathbf{u}_{i}\right)>\frac{1}{2}\right)=l_{i}\right)=\frac{1}{N_{\text {test }}} \sum_{i \in I_{\text {test }}} \widehat{\operatorname{acc}}_{i}
$$

where $I_{\text {test }}=\left\{i:\left(\mathbf{u}_{i}, l_{i}\right) \in D_{\text {test }}\right\}$.

(5) Compute a $p$-value based on the asymptotically normal null distribution of acc:

$$
\hat{p}=\operatorname{Pr}\left(\widehat{a c c}^{\prime} \geq \widehat{a c c} \mid H_{0}\right)=1-\Phi\left(\frac{\widehat{a c c}-1 / 2}{\sqrt{\frac{1}{4 N_{\text {test }}}}}\right)
$$

where $\Phi(\cdot)$ denotes the standard normal cumulative distribution function.

To derive Equation (3.2), notice that $a c c_{i} \sim \operatorname{Bernoulli}\left(a c c_{i} \mid p_{i}\right)$ where $p_{i}=1 / 2$ is the probability of correctly classifying some $\mathbf{u}_{i}$ in the test set when $H_{0}$ is true. In this setting, Equation (3.2) follows from the fact that

$$
N_{\text {test }} a c c \sim \operatorname{Binomial}\left(N_{\text {test }} a c c \mid N_{\text {test }}, \frac{1}{2}\right) \approx \mathscr{N}\left(N_{\text {test }} a c c \mid \frac{N_{\text {test }}}{2}, \frac{N_{\text {test }}}{4}\right)
$$

as $N_{\text {test }} \rightarrow \infty$.

C2STs provide a variety of interpretable numbers that complement the use of $p$-values:

(a) Taking $\hat{f}\left(\mathbf{u}_{i}\right)$ as an estimate of the conditional probability $\operatorname{Pr}\left(l_{i}=1 \mid \mathbf{u}_{i}\right)$ for $i \in I_{\text {test }}$, I can determine which item response patterns were labeled correctly or incorrectly as well as how confident $\hat{f}$ was in each decision. This approach provides a way to evaluate which observed response patterns are discrepant from the fitted IFA model.

(b) I can interpret the fitted classifier $\hat{f}$ to determine which items were most useful for distinguishing between real and synthetic distributions (e.g., by interpreting a logistic regression classifier's coefficients).

(c) I can interpret the test statistic $\widehat{a c c}$ as the percentage of item response patterns that were 
correctly distinguished between the real and synthetic distributions.

The values described in (a), (b), and (c) correspond to measures of person fit, piece-wise fit, and overall model fit, respectively. C2STs may therefore be viewed as uniting these different kinds of fit measures under a single framework.

C2STs are closely related to classical two-sample tests including the $t$-test for the difference in means of two samples (Student 1908), the Wilcoxon-Mann-Whitney test for the difference in rank means of two samples (Mann \& Whitney 1947; Wilcoxon 1945), and the Kolmogorov-Smirnov test for the difference in empirical cumulative distributions of two samples (Kolmogorov 1933; Smirnov 1939), although these classical tests are less statistically efficient in the multivariate setting. C2STs are also related to modern multivariate kernel two-sample tests (K2STs) including the maximum mean discrepancy test (Gretton, Borgwardt, Rasch, Schölkopf, \& Smola 2012) and the mean embedding test (Chwialkowski, Ramdas, Sejdinovic, \& Gretton 2015; Jitkrittum, Szabó, Chwialkowski, \& Gretton 2016), both of which test for differences in the empirical kernel mean embeddings of two samples. However, depending on one's choice for $f$, C2STs typically improve upon K2STs by requiring less manual engineering of the input data and by providing more interpretable results.

\subsection{Approximate C2STs}

The C2STs described above are exact in the sense that they test the null hypothesis that the real distribution $\mathbb{P}$ and the synthetic distribution $\hat{\mathbb{P}}$ are exactly equal. In general, however, it is unlikely that any specified IFA model will exactly capture the data generating mechanism such that $\mathbb{P}=\hat{\mathbb{P}}$ (e.g., Cudeck \& Henly 1991; Maccallum \& Tucker 1991). I therefore propose a more realistic approximate C2ST for which I assume $\mathbb{P} \neq \hat{\mathbb{P}}$ and I test $H_{0}: a c c=1 / 2+\delta$ against $H_{1}:$ acc $>$ $1 / 2+\delta$ where $\delta \in(0,1 / 2)$ is a pre-specified value representing the degree of model error viewed as tolerable by the user. The approximate $\mathrm{C} 2 \mathrm{ST}$ is not a test of exact GOF because it does not test whether the hypothesized IFA model exactly captures the data generating mechanism. Instead, the approximate $\mathrm{C} 2 \mathrm{ST}$ is a test of approximate GOF wherein the user asserts that an IFA model that fits the data "well enough" should be capable of synthesizing item response patterns that can only 
be distinguished from real item response patterns around $100 \delta \%$ of the time.

I now derive the null distribution of $a c c$ for the approximate C2ST. Since acc $=1 / 2+\delta>1 / 2$ under $H_{0}$, Equation (3.1) implies that $a c c_{i}=1 / 2+\delta_{i} \geq 1 / 2$ for $i \in I_{\text {test }}$ where $\delta_{i} \in[0,1 / 2]$ and $\delta=N_{\text {test }}^{-1} \sum_{i \in I_{\text {test }}} \delta_{i}$. In this setting, the $a c c_{i}$ are independent but not identically distributed Bernoulli random variables with success probabilities $p_{i}=1 / 2+\delta_{i} . N_{\text {test }}$ acc therefore follows a Poisson binomial distribution, which I follow Ehm (1991) in approximating as

$$
N_{\text {test }} a c c \sim \operatorname{Binomial}\left(N_{\text {test }} a c c \mid N_{\text {test }}, \bar{p}\right) \approx \mathscr{N}\left(N_{\text {test }} a c c \mid N_{\text {test }} \bar{p}, N_{\text {test }} \bar{p}(1-\bar{p})\right)
$$

as $N_{\text {test }} \rightarrow \infty$ where $\bar{p}=N_{\text {test }}^{-1} \sum_{i \in I_{\text {test }}} p_{i}=1 / 2+N_{\text {test }}^{-1} \sum_{i \in I_{\text {test }}} \delta_{i}=1 / 2+\delta$. It follows that

$$
a c c \sim \mathscr{N}\left(a c c \mid \frac{1}{2}+\delta, \frac{\frac{1}{4}-\delta^{2}}{N_{\text {test }}}\right) .
$$

To derive the alternative distribution of $a c c$, notice that under $H_{1}$ I have $a c c=1 / 2+\delta+\varepsilon>1 / 2$ where the effect size $\varepsilon \in(0,1 / 2-\delta)$ is the magnitude of the difference between acc under $H_{0}$ and acc under $H_{1}$. Equation (3.1) implies that $a c c_{i}=1 / 2+\delta_{i}+\varepsilon_{i}$ for $i \in I_{\text {test }}$ where $\delta_{i} \in[0,1 / 2]$, $\varepsilon_{i} \in\left[0,1 / 2-\delta_{i}\right], \delta=N_{\text {test }}^{-1} \sum_{i \in I_{\text {test }}} \delta_{i}$, and $\varepsilon=N_{\text {test }}^{-1} \sum_{i \in I_{\text {test }}} \varepsilon_{i}$. Then by a similar argument to the one given in the previous paragraph, I can obtain

$$
a c c \sim \mathscr{N}\left(a c c \mid \frac{1}{2}+\delta+\varepsilon, \frac{\frac{1}{4}-\delta^{2}-2 \delta \varepsilon-\varepsilon^{2}}{N_{\text {test }}}\right)
$$

as $N_{\text {test }} \rightarrow \infty$.

I now analyze the approximate C2ST's power (i.e., the probability of correctly rejecting $H_{0}$ when $H_{0}$ is false) by proving the following theorem.

Theorem 1. Let $\alpha \in[0,1]$ be the user-defined significance level (i.e., the probability of incorrectly rejecting $H_{0}$ when $H_{0}$ is true). Suppose the null and alternative distributions of acc are given by equations 3.5 and 3.6, respectively. Then the power of the approximate C2ST is approximately 
given by

$$
\operatorname{power}\left(\alpha, N_{\text {test }}, \delta, \varepsilon\right)=\Phi\left(\frac{\varepsilon \sqrt{N_{\text {test }}}-\sqrt{\frac{1}{4}-\delta^{2}} \Phi^{-1}(1-\alpha)}{\sqrt{\frac{1}{4}-\delta^{2}-2 \delta \varepsilon-\varepsilon^{2}}}\right) .
$$

Proof. At significance level $\alpha$, the decision threshold for acc is

$$
z_{\alpha}=\frac{1}{2}+\delta+\sqrt{\frac{\frac{1}{4}-\delta^{2}}{N_{\text {test }}}} \Phi^{-1}(1-\alpha)
$$

When $a c c<z_{\alpha}$, we accept $H_{0}$. The probability of making a type II error (i.e., of incorrectly accepting $H_{0}$ when $H_{0}$ is false) is

$$
\begin{aligned}
\mathscr{N}\left(a c c \mid \frac{1}{2}+\delta+\varepsilon, \frac{\left.\operatorname{Pr}_{\frac{1}{4}-\delta^{2}-2 \delta \varepsilon-\varepsilon^{2}}^{N_{\text {test }}}\right)}{\left(a c c<z_{\alpha}\right)}\right. & =\operatorname{Pr}_{\mathscr{N}\left(a c c^{\prime} \mid 0 \frac{\frac{1}{4}-\delta^{2}-2 \delta \varepsilon-\varepsilon^{2}}{N_{\text {test }}}\right)}\left(a c c^{\prime}<\sqrt{\frac{\frac{1}{4}-\delta^{2}}{N_{\text {test }}}} \Phi^{-1}(1-\alpha)-\varepsilon\right) \\
& =\Phi\left(\sqrt{\frac{N_{\text {test }}}{\frac{1}{4}-\delta^{2}-2 \delta \varepsilon-\varepsilon^{2}}}\left(\sqrt{\frac{\frac{1}{4}-\delta^{2}}{N_{\text {test }}}} \Phi^{-1}(1-\alpha)-\varepsilon\right)\right) \\
& =\Phi\left(\frac{\sqrt{\frac{1}{4}-\delta^{2}} \Phi^{-1}(1-\alpha)-\varepsilon \sqrt{N_{\text {test }}}}{\sqrt{\frac{1}{4}-\delta^{2}-2 \delta \varepsilon-\varepsilon^{2}}}\right) .
\end{aligned}
$$

The power of the approximate $\mathrm{C} 2 \mathrm{ST}$ is therefore

$$
\begin{aligned}
\operatorname{power}\left(\alpha, N_{\text {test }}, \delta, \varepsilon\right) & =1-\Phi\left(\frac{\sqrt{\frac{1}{4}-\delta^{2}} \Phi^{-1}(1-\alpha)-\varepsilon \sqrt{N_{\text {test }}}}{\sqrt{\frac{1}{4}-\delta^{2}-2 \delta \varepsilon-\varepsilon^{2}}}\right) \\
& =\Phi\left(\frac{\varepsilon \sqrt{N_{\text {test }}}-\sqrt{\frac{1}{4}-\delta^{2}} \Phi^{-1}(1-\alpha)}{\sqrt{\frac{1}{4}-\delta^{2}-2 \delta \varepsilon-\varepsilon^{2}}}\right)
\end{aligned}
$$

Remark 1. The (approximate) power of the exact C2ST was derived by Lopez-Paz and Oquab (2017, Theorem 1) and can be alternately be derived by setting $\delta=0$ in the power formula in Theorem 1. 
Theorem 1 provides an important insight: maximizing the approximate C2ST's power involves trading off between maximizing the test set size $N_{\text {test }}$ and choosing a classifier $f$ that maximizes the effect size $\varepsilon$ (equivalently, that maximizes the test set accuracy $a c c=1 / 2+\delta+\varepsilon$ ). To illustrate this trade-off, notice that while relatively inflexible classifiers (e.g., logistic regression) often obtain low test set accuracy because they cannot capture complex nonlinear relationships (i.e., they decrease $\varepsilon$ ), these classifiers only require small training data sets (i.e., they increase $N_{\text {test }}$ ). Conversely, while flexible classifiers (e.g., NNs) may obtain high test set accuracy by capturing complex nonlinear relationships (i.e., they increase $\varepsilon$ ), these classifiers require large training data sets (i.e., they decrease $N_{\text {test }}$ ). Lopez-Paz and Oquab (2017) note that this trade-off is analogous to the well known bias-variance trade-off in statistics and machine learning (e.g., Yarkoni \& Westfall 2017). In this thesis, I empirically investigate this tradeoff by using two different classifiers (i.e., a $k$-nearest neighbors classifier and an $\mathrm{NN}$ classifier) that may require different training set sizes to obtain optimal test set accuracy. I also follow Jitkrittum et al. (2016) and Lopez-Paz and Oquab (2017) in setting $N_{\text {train }}=N_{\text {test }}=N$, which often achieves high power in practice and would achieve maximum power if $\mathbb{P}$ and $\hat{\mathbb{P}}$ differed only in means.

\subsection{C2ST-Based Relative Fit Index}

The C2STs described above measure how well the proposed IFA model reproduces the observed data (i.e., the model's absolute GOF). An alternative approach that is often used in SEM is to calculate a relative fit index (RFI) that measures the proportional improvement in fit obtained by moving to the proposed model from a more restrictive baseline model (e.g., Bentler 1990; Bentler \& Bonett 1980; Bollen 1989a; L. R. Tucker \& Lewis 1973). A typical baseline model posits that the observed variables are mutually independent (i.e., there are no common latent factors underlying the data; Bentler \& Bonett 1980) and serves as a contrasting point of reference to a model that perfectly reproduces the observed data (i.e., a saturated model; e.g., Bentler 1995; Steiger 1980).

Consider a zero-factor baseline model where $P=0$ and $\boldsymbol{\theta}_{j}=\boldsymbol{\alpha}_{j}$ for $j=1, \ldots, J$. In this case, each respondent's response probability $\pi_{i, j, k}$ evaluated at the maximum likelihood estimate of $\boldsymbol{\theta}$ can be shown to be the observed proportion of respondents choosing response category $k$ for item 
$j$, which I write as $\hat{\pi}_{j, k}=N^{-1} \sum_{i=1}^{N} \mathbb{1}\left(x_{i, j}=k\right)$. I can therefore conduct a C2ST for this baseline model using $N$ synthetic samples drawn from a multinomial distribution with $K$ cells, trial size 1 , and cell probabilities $\hat{\pi}_{j, k}$. Let $\widehat{a c c}_{\text {prop }}$ and $\widehat{a c c}_{\text {base }}$ denote the test set accuracies for the proposed model and for the baseline model, respectively, and let $\hat{f}_{\text {prop }}$ and $\hat{f}_{\text {base }}$ denote the corresponding fitted classifiers. I propose the following C2ST-based RFI:

$$
\mathrm{C} 2 \mathrm{ST}-\mathrm{RFI}=1-\frac{M_{\text {prop }}}{M_{\text {base }}} \cdot \frac{\Delta_{\text {prop }}}{\Delta_{\text {base }}}
$$

where $\Delta_{\text {prop }}=\widehat{a c c}_{\text {prop }}-1 / 2, \Delta_{\text {base }}=\widehat{a c c}_{\text {base }}-1 / 2, M_{\text {prop }}$ is the number of fitted parameters in the proposed model, and $M_{\text {base }}$ is the number of fitted parameters in the baseline model. The ratio $\Delta_{\text {prop }} / \Delta_{\text {base }}$ is a measure of the proportional change in misfit obtained by moving to the proposed model from the baseline model, while the ratio $M_{\text {prop }} / M_{\text {base }}$ is a penalty that increases with the number of fitted parameters in the proposed model (i.e., it rewards parsimony in the proposed model).

I motivate my definition of C2ST-RFI by considering the fit index's behavior in the typical setting where the baseline model obtains less-than-perfect fit (i.e., when $\widehat{a c c}_{\text {base }}>1 / 2$ ). In the common scenario that the proposed model fits the same or better than the baseline model (i.e., when $\widehat{a c c}_{\text {prop }}$ varies between $\widehat{a c c}_{\text {base }}$ and $1 / 2$ ), C2ST-RFI varies between $1-M_{\text {prop }} / M_{\text {base }}$ and one with values closer to one indicating better fit. C2ST-RFI is much larger than one when $\widehat{a c c}_{\text {prop }}$ is much smaller than $1 / 2$, which may occur when proposed model fits the data well but $\hat{f}_{\text {prop }}$ has overfitted the training data. In the infrequent event that the proposed model fits worse than the baseline model (i.e., when $\widehat{a c c}_{\text {prop }}>\widehat{a c c}_{\text {base }}$ ), C2ST-RFI is smaller than $1-M_{\text {prop }} / M_{\text {base }}$. C2ST-RFI therefore behaves somewhat analogously to other nonnormed fit indices such as the Tucker-Lewis Index (L. R. Tucker \& Lewis 1973) that distinguish between less-than-perfect model fit, perfect model fit, and overfitting by being smaller than one in the first case, close to one in the second case, and much larger than one in the third case.

I next consider the effect of sample size on C2ST-RFI. Bollen (1989a) notes that GOF indices may be influenced by sample size either (a) when $N$ enters the calculation of the index or (b) when 
the mean of the sampling distribution of the index is related to $N$. For (a), notice that although both $\widehat{a c c}_{\text {prop }}$ and $\widehat{a c c}_{\text {base }}$ include factors of $N_{\text {test }}^{-1}$, these factors cancel in the ratio $\Delta_{\text {prop }} / \Delta_{\text {base }}$, indicating that (a) does not hold. For (b), notice that when $N$ is very small, both $\hat{f}_{\text {prop }}$ and $\hat{f}_{\text {base }}$ may fail to capture any relationships in the training data. In this case, both $\widehat{a c c}_{\text {prop }}$ and $\widehat{a c c}_{\text {base }}$ will be close to $1 / 2$ and C2ST-RFI will be close to $1-M_{\text {prop }} / M_{\text {base. }}$ As $N$ increases, $\hat{f}_{\text {prop }}$ and $\hat{f}_{\text {base }}$ should capture any relationships in the training data and $\widehat{a c c}_{\text {prop }}$ and $\widehat{a c c}_{\text {base }}$ should come close to their population values, suggesting that (b) holds. I provide empirical evidence that the mean of the sampling distribution of C2ST-RFI depends on $N$ in Chapter 4. Specifically, I observe that C2STRFI gives a less optimistic assessment of fit as $N$ increases. This phenomenon is analogous to the approximate C2ST's power increasing with $N$ and is likely of little concern for moderate- to large-scale applications.

I also consider the probability limit of the proposed fit index. Assume that $\operatorname{plim}_{N \rightarrow \infty}\left(a c_{\text {prop }}\right)=$ $1 / 2+\delta$ and $\operatorname{plim}_{N \rightarrow \infty}\left(a c c_{\text {base }}\right)=1 / 2+\delta+\varepsilon$ where $\delta \in[0,1 / 2]$ and $\varepsilon \in[0,1 / 2-\delta]$. This assumption is mild and states that the accuracies obtained by $\hat{f}_{\text {prop }}$ and $\hat{f}_{\text {base }}$ should come close to specific constants as $N$ grows large, with $\hat{f}_{\text {prop }}$ obtaining the same or better accuracy than $\hat{f}_{\text {base }} \cdot{ }^{1}$ Then $\operatorname{plim}_{N \rightarrow \infty}(\mathrm{C} 2 \mathrm{ST}-\mathrm{RFI})=1-\left[M_{\text {prop }} / M_{\text {base }}\right] \cdot[\delta /(\delta+\varepsilon)]=c$ where $c$ is a constant which equals one when the proposed model is correct (i.e., when $\delta=0$ ) and is smaller than one otherwise.

Finally, I comment on how to interpret numerical values of C2ST-RFIs. In practice, cutoff values are used to interpret fit indices and thereby evaluate model fit. The development of adequate "rules of thumb" cutoff criteria that cover a wide range of realistic data and model conditions requires extensive empirical study (e.g., Hu \& Bentler 1999) and is beyond the scope of this thesis. Based on limited initial experimentation, however, I consider a provisory cutoff of C2ST-RFI $>0.9$ to indicate good fit. I empirically investigate the performance of this cutoff in Chapter 4.

\footnotetext{
${ }^{1}$ A similar assumption that $\operatorname{plim}_{N, J \rightarrow \infty}($ acc $)=1 / 2+\delta$ for some $\delta>0$ is used by Kim et al. (2021) to prove that C2STs are consistent (i.e., have power approaching one) in the high-dimensional setting where both $N$ and $J$ tend to infinity.
} 


\subsection{Summary}

I began this chapter by formally describing C2STs as well as their application to exact GOF assessment for confirmatory IFA models. I subsequently extended C2STs in ways that make them better suited to real-world applications where the proposed model is not the exact data-generating model: I derived an approximate C2ST wherein the user specifies the proportion of model error they deem acceptable as well as a C2ST-based RFI that parallels the fit indices used in SEM. These developments were primarily theoretical and will be investigated experimentally in the following chapter. 


\section{IMPLEMENTATION AND EXPERIMENTS}

\subsection{Implementation}

I-WAVE is programmed using the machine learning library PyTorch (Version 1.1.6 Paszke et al. 2017). Although PyTorch supports GPU computing to accelerate fitting, I follow Urban and Bauer (2021) in opting for CPU computing to enable fairer comparisons with other methods and to assess performance using hardware that is more typically available to social scientists. Experiments are conducted on a computer with a $2.8 \mathrm{GHz}$ Intel Core i7 CPU and $16 \mathrm{~GB}$ of RAM. Code to reproduce all experiments is readily available at https://github.com/cjurban/ MachineLearningConfirmatoryIFA.

Optimization and hyperparameter tuning for I-WAVE in the exploratory setting are discussed in detail by Urban and Bauer (2021, Section 5). I follow their implementation almost exactly, although I adapt their factor loadings initialization procedure to the confirmatory setting as follows:

$$
\beta_{j, p} \sim \text { Uniform }\left(\beta_{j, p} \mid-\sqrt{\frac{6}{M_{p}+P}}, \sqrt{\frac{6}{M_{p}+P}}\right)
$$

for $j=1, \ldots, J$ and $p=1, \ldots, P$ where $M_{p}$ is the number of nonzero loadings parameters for factor p. This strategy is based on Xavier initialization (Glorot \& Bengio 2010) and aims stabilize fitting while taking into account user-defined zero constraints on the factor loadings. An additional detail that must be addressed in the confirmatory setting is initializing the hyperspherical parameterization $\Theta$ of the factor correlation matrix $\boldsymbol{\Sigma}$. To ensure that $\boldsymbol{\Sigma}$ is well-conditioned at the start of fitting, I choose $\boldsymbol{\Theta}$ such that $\boldsymbol{\Sigma}=\mathbf{I}_{P}$ by setting $\vartheta_{p, p^{\prime}}=\pi / 2$ for $p=1, \ldots, P, p^{\prime}=1, \ldots, p-1$.

Following Lopez-Paz and Oquab (2017), I investigate two classifiers for C2ST analyses: a $k$ nearest neighbors (KNN) classifier (Fix \& Hodges 1951) and an NN classifier. ${ }^{1}$ Both classifiers are

${ }^{1}$ I initially also investigated a regularized logistic regression classifier but found that it performed very poorly relative to KNNs and NNs. 
implemented via the Python package scikit-learn (Version 0.23.1; Pedregosa et al. 2011) and have different hyperparameter settings, which I discuss in turn. For each test set observation $\mathbf{u}_{i} \in \mathscr{X}$, let $I_{i}$ collect the indices of the $k$ training set observations that are closest to $\mathbf{u}_{i}$ under some distance metric $d: \mathscr{X} \times \mathscr{X} \rightarrow[0, \infty)$. KNNs set the predicted class $\hat{l}_{i}$ for $\mathbf{u}_{i}$ to the most frequent observed class for the $k$ closest training set observations, that is, $\hat{l}_{i}=\mathbb{1}\left(k^{-1} \sum_{i^{\prime} \in I_{i}} l_{i^{\prime}}>1 / 2\right)$. In this work, I use the Hamming distance metric $d\left(\mathbf{u}_{i}, \mathbf{u}_{i^{\prime}}\right)=J^{-1} \sum_{j=1}^{J} \mathbb{1}\left(u_{i, j} \neq u_{i^{\prime}, j}\right)$, which returns the proportion of unequal responses between $\mathbf{u}_{i}$ and $\mathbf{u}_{i^{\prime}}$. I also set $k=\left\lfloor\sqrt{N_{\text {test }}}\right\rfloor$, which often performs adequately in practice (Duda, Hart, \& Stork 2001, Chapter 4).

Although KNNs are flexible and often require very little tuning, they typically obtain worse predictive accuracy as $J$ increases and are computationally intensive when the training set size $N_{\text {train }}$ is large (Hastie, Tibshirani, \& Friedman 2009, Chapter 13). NNs overcome these issues in that their predictive accuracy suffers relatively less as $J$ increases and their computational efficiency does not depend on $N_{\text {train }}$ when they are fitted via stochastic gradient methods (Bottou, Curtis, \& Nocedal 2018), although they often require more tuning than KNNs to obtain optimal predictive accuracy. I set most NN hyperparameters to the scikit-learn package defaults, which performed well in all experiments. To mitigate overfitting in small data sets, I (a) stop fitting if the default convergence criterion is not obtained within $\left\lfloor 10000 \cdot 200 / N_{\text {test }}\right\rfloor$ stochastic gradient steps and (b) apply weight decay (i.e., an $L_{2}$ norm penalty on the $\mathrm{NN}$ regression weights) where the optimal weight decay hyperparameter is selected from $\left\{10^{n}: n \in\{-1,-1 / 2,0,1 / 2,1\}\right\}$ using a held-out validation set consisting of $25 \%$ of the training set observations (for details, see Hastie et al. 2009, Chapters 7 and 11).

Since neither KNNs nor NNs are directly interpretable, I use a permutation importance (PI) method to interpret which items are most useful for distinguishing between real and synthetic distributions (Breiman 2001; Fisher, Rudin, \& Dominici 2019). PI can be used to interpret any fitted classifier $\hat{f}$, even when $\hat{f}$ itself is not directly interpretable. Let $\mathbf{U}$ be an $N \times J$ matrix whose $i^{\text {th }}$ row is $\mathbf{u}_{i}^{\top}$ for $i \in I_{\text {test. }}$. For each item $j=1, \ldots, J$ and each repetition $t=1, \ldots, T$, shuffle the $j^{\text {th }}$ column of $\mathbf{U}$ to generate a corrupted matrix $\widetilde{\mathbf{U}}_{j, t}$ whose $i^{\text {th }}$ row is $\widetilde{\mathbf{u}}_{i, j, t}^{\top}$. The PI for item $j$ is defined 
as the average decrease in test set accuracy for $\hat{f}$ when the responses to item $j$ are shuffled:

$$
\text { importance }_{j}=\widehat{\operatorname{acc}}-\frac{1}{T} \sum_{t=1}^{T} \frac{1}{N_{\text {test }}} \sum_{i \in I_{\text {test }}} \mathbb{1}\left(\mathbb{1}\left(\hat{f}\left(\widetilde{\mathbf{u}}_{i, j, t}\right)>\frac{1}{2}\right)=l_{i}\right)=\widehat{a c c}-\frac{1}{T} \sum_{t=1}^{T} \widetilde{a c c}_{j, t} \text {. }
$$

Since the shuffling procedure breaks the relationship between item $j$ and the class label, importance ${ }_{j}$ measures how much $\hat{f}$ depends on item $j$. PI may be viewed as a piece-wise fit assessment method wherein each importance ${ }_{j}$ measures how well the proposed IFA model fits item $j$, with larger importance $_{j}$ indicating worse fit. I set the number of repetitions to $T=5$ for all experiments.

\subsection{Empirical Example}

I demonstrate the proposed methods' computational efficiency in the large-scale setting and obtain data generating parameters for simulation studies by analyzing 1015342 responses to the 50-item International Personality Item Pool five-factor model (IPIP-FFM; Goldberg 1999), the same data considered by Urban and Bauer (2021) for exploratory IFA using I-WAVE. The IPIPFFM is designed to measure the Big Five personality factors of openness, conscientiousness, extraversion, agreeableness, and emotional stability, making this data well suited to a more confirmatory approach. Each factor is measured by 10 five-category items anchored by "Disagree" (1), "Neutral" (3), and "Agree" (5). The data were downloaded from the Open-Source Psychometrics Project (https: / / openpsychometrics.org/) and pre-processed following Urban and Bauer (2021, Section 6.1), resulting in an analytic sample containing $N=515708$ responses. Reverse worded items were recoded so that the highest numerical response category indicated a high level of the corresponding factor.

\subsubsection{A Five-Factor Model}

I used I-WAVE to fit a five-factor confirmatory IFA model with correlated factors to the IPIPFFM data. Optimization and inference model hyperparameters were set to the values used by Urban and Bauer (2021, Section 6.1). I set the number of IW samples to $R=5$ based on Urban and Bauer's (2021) finding that this value performs well in practice. I fitted the data set 10 times to investigate whether parameter estimation and GOF assessment results were stable across random starts. 
Table 4.1: Factor Loadings for IPIP-FFM Data Set

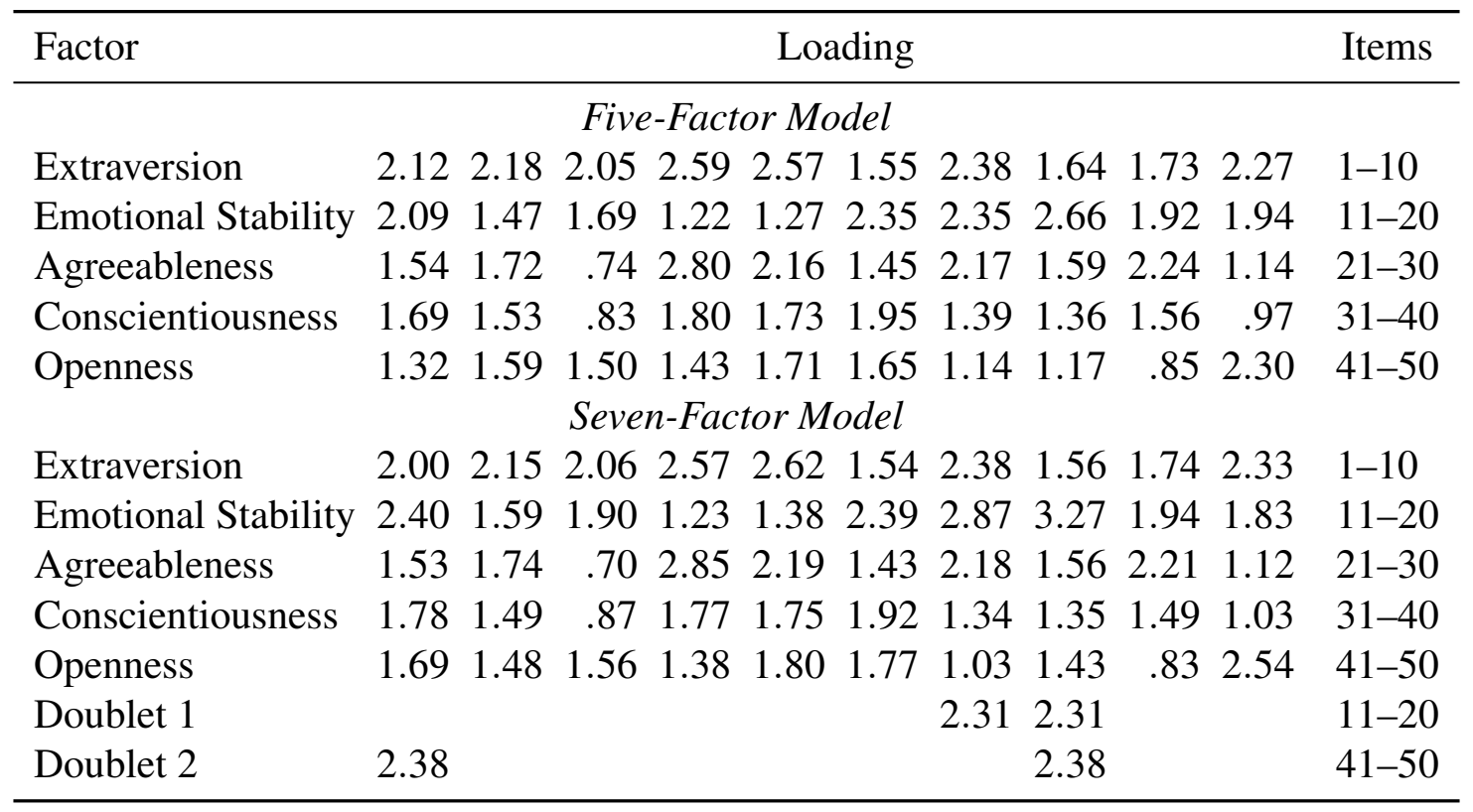

Factor loadings and correlations from the fitted model that attained the highest IW-ELBO across random starts - henceforth called the reference model - are reported in Tables 4.1 and 4.2, respectively. All loadings were positive, which fit with the confirmatory design of the measurement scale. Factor correlations aligned with the typical finding that emotional stability is negatively correlated with the other factors. The mean fitting time was 198 seconds $(S D=56$ seconds), which is quite fast given the large sample size. Relative to the reference model, mean loadings root-mean-square error (RMSE) was $0.031(S D=0.017)$, mean intercepts RMSE was $0.046(S D=0.018)$, and mean factor correlation RMSE was $0.027(S D=0.010)$, suggesting that fitting was fairly stable. ${ }^{2}$

I assessed overall fit for the proposed five-factor model (FFM) across random starts using KNNand NN-based exact and approximate C2STs. The large sample size required minor modifications to how the classifiers were implemented. Specifically, I fitted the KNNs using a random subsample of $2.5 \%$ of the training set observations to ensure that fitting was not computationally intensive,

\footnotetext{
${ }^{2}$ I treat the model attaining the highest IW-ELBO as ground truth in line with how an optimal solution is often selected from multiple random starts for mixture models (e.g., Biernacki, Celeux, \& Govaert 2003). Were I to alternatively treat the mean parameter estimates across random starts as ground truth, the corresponding variability estimates would be smaller than those reported here, although this approach would downplay the impact of possible local maxima on parameter estimate stability.
} 
Table 4.2: Factor Correlations for IPIP-FFM Data Set

\begin{tabular}{|c|c|c|c|c|c|c|c|}
\hline \multirow[b]{2}{*}{ Factor } & \multicolumn{7}{|c|}{ Factor } \\
\hline & 1 & 2 & 3 & 4 & 5 & 6 & 7 \\
\hline \multicolumn{8}{|c|}{ Five-Factor Model } \\
\hline 1. Extraversion & 1.00 & & & & & & \\
\hline 2. Emotional Stability & -.23 & 1.00 & & & & & \\
\hline 3. Agreeableness & .37 & .01 & 1.00 & & & & \\
\hline 4. Conscientiousness & .10 & -.28 & .08 & 1.00 & & & \\
\hline 5. Openness & .21 & -.10 & .18 & .01 & 1.00 & & \\
\hline \multicolumn{8}{|c|}{ Seven-Factor Model } \\
\hline 1. Extraversion & 1.00 & & & & & & \\
\hline 2. Emotional Stability & -.26 & 1.00 & & & & & \\
\hline 3. Agreeableness & .38 & .02 & 1.00 & & & & \\
\hline 4. Conscientiousness & .09 & -.27 & .11 & 1.00 & & & \\
\hline 5. Openness & .26 & -.15 & .20 & .06 & 1.00 & & \\
\hline 6. Doublet 1 & .00 & .00 & .00 & .00 & .00 & 1.00 & \\
\hline 7. Doublet 2 & .00 & .00 & .00 & .00 & .00 & .00 & 1.00 \\
\hline
\end{tabular}

and I did not apply weight decay to the NNs given that large sample sizes often mitigate overfitting. For approximate C2STs, I set $\delta=0.025$ to test $H_{0}: a c c=0.525$ against $H_{1}:$ acc $>0.525$, which amounts to testing whether the proposed FFM is capable of synthesizing item response patterns that can only be distinguished from real item response patterns $52.5 \%$ of the time (i.e., at slightly better than chance). C2ST results are presented in Table 4.3. The fitted KNNs obtained much lower test set accuracies than the fitted NNs ( $M=0.67$ for KNNs vs. $M=0.83$ for NNs), echoing the typical finding that NNs outperform KNNs when $J$ is large. All KNN- and NN-based C2STs rejected $H_{0}$ at signficance level $\alpha=0.05(\hat{p}<0.0001)$, suggesting that the FFM did not fit the data "well enough". Importantly, all tests were fast: accounting for both sampling synthetic data and fitting the classifier, KNN-based C2STs took around 20 seconds and NN-based C2STs took around 15 seconds. 
Table 4.3: Classifier Two-Sample Test Results for IPIP-FFM Data Set

\begin{tabular}{|c|c|c|c|c|c|c|}
\hline & \multicolumn{2}{|c|}{ Baseline Model } & \multicolumn{2}{|c|}{ Five-Factor Model } & \multicolumn{2}{|c|}{ Seven-Factor Model } \\
\hline & $M$ & $S D$ & $M$ & $S D$ & $M$ & $S D$ \\
\hline Sampling Time & 6.8 & $<.1$ & 8.7 & .4 & 8.7 & .7 \\
\hline \multicolumn{7}{|l|}{ KNN } \\
\hline Fitting Time & 11.0 & .7 & 11.8 & 2.2 & 11.6 & .7 \\
\hline Test Set Accuracy & .65 & .01 & .67 & .01 & .66 & .01 \\
\hline C2ST-RFI & & & -.49 & .22 & -.46 & .21 \\
\hline \multicolumn{7}{|l|}{$\mathrm{NN}$} \\
\hline Fitting Time & 6.5 & $<.1$ & 6.6 & .3 & 6.6 & $<.1$ \\
\hline Test Set Accuracy & .96 & $<.01$ & .83 & $<.01$ & .81 & $<.01$ \\
\hline C2ST-RFI & & & .05 & .02 & .10 & .01 \\
\hline
\end{tabular}

Note. "Sampling Time" refers to time required to sample synthetic data from the model. All times are given in seconds.

I next assessed piece-wise fit by computing PIs for fitted classifiers accross random starts, which are displayed in Figure 4.1a. The median NN importances are clearly much larger than the median KNN importances, likely due to the NNs having obtained higher test set accuracies. Further, the NNs rely heavily on five specific items - items 17,18 , and 20 , which measure emotional stability, and items 41 and 48, which measure openness - whereas the KNNs rely fairly evenly on all the items. This finding suggests that the NNs captured relationships between these five items and the class label that the KNNs did not, likely contributing to the NNs' superior performance. The NN results in particular suggest that the FFM's overall lack of fit is most heavily influenced by its piece-wise lack of fit to items $17,18,20,41$, and 48.

Although the above results suggest that the FFM did not fit the data well in an absolute sense, similar findings might be expected for nearly any a priori model. It would therefore be useful 

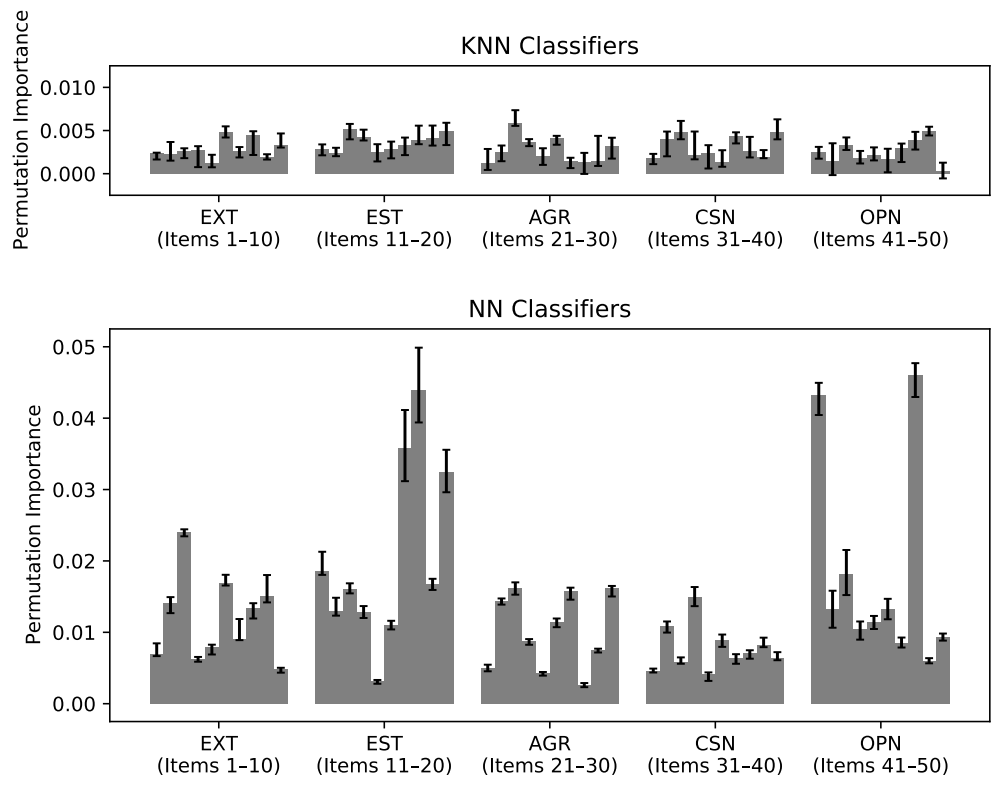

(a) Five-factor model.
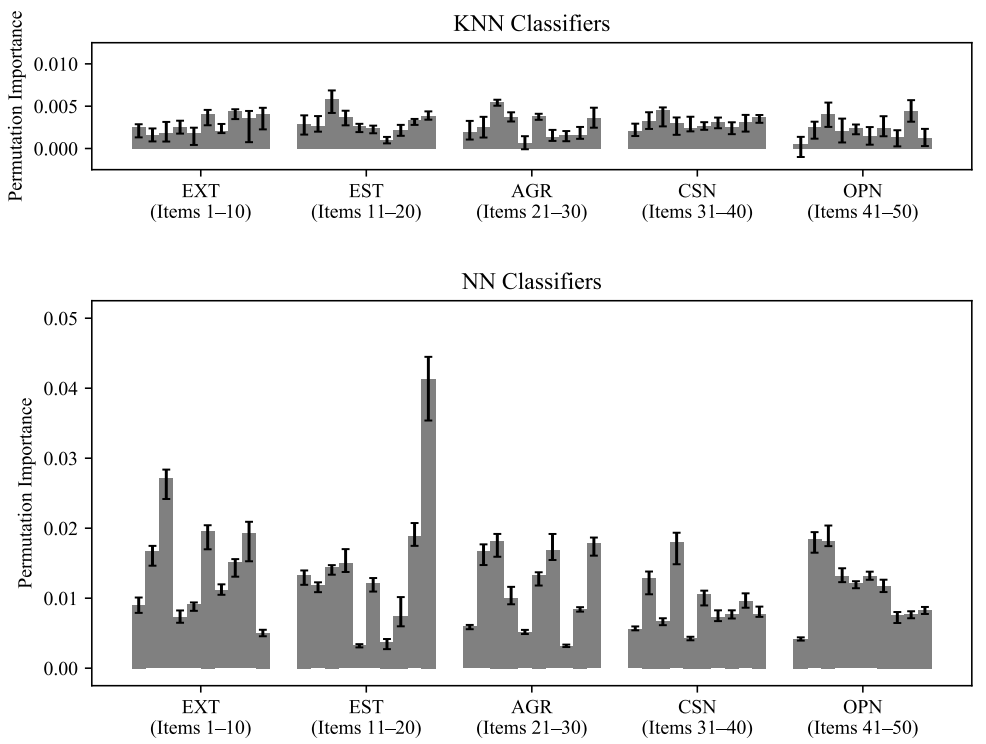

(b) Seven-factor model.

Figure 4.1: Permutation importances for each IPIP-FFM item across 10 random starts. Items are grouped by their corresponding factor. Bar heights indicate medians, while error bars indicate $25 \%$ and $75 \%$ quantiles. Hatched bars indicate the five poorest fitting items for the five-factor model. $\mathrm{EXT}=$ extraversion, $\mathrm{EST}=$ emotional stability, $\mathrm{AGR}=$ agreeableness, $\mathrm{CON}=$ conscientiousness, $\mathrm{OPN}=$ openness. 
Table 4.4: Wordings for Five Poorest Fitting IPIP-FFM Items

\begin{tabular}{ll}
\hline Item & \multicolumn{1}{c}{ Wording } \\
\hline 17 & I change my mood a lot. \\
18 & I have frequent mood swings. \\
20 & I often feel blue. \\
41 & I have a rich vocabulary. \\
48 & I use difficult words. \\
\hline
\end{tabular}

to assess the FFM's value in a relative sense by contrasting it with a baseline model. To this end, I computed C2ST-RFIs for the fitted classifiers across random starts. As shown in Table 4.3, KNNs obtained much lower baseline model test set accuracies than NNs $(M=0.65$ for KNNs vs. $M=0.96$ for NNs). The KNN results suggest that the proposed FFM and the baseline zerofactor model had comparable fit, while the NN results suggest that the FFM fit the data better than the baseline model. This finding was reflected in the C2ST-RFIs, which were much lower for KNNs than for NNs ( $M=-0.49$ for KNNs vs. $M=0.05$ for NNs). No C2ST-RFIs exceeded the provisory cutoff of 0.9 suggested as an indicator of good fit. The additional RFI computations were also fast, taking at most around 18 seconds or less.

\subsubsection{A Seven-Factor Model}

I investigated the five poorest fitting items flagged by PIs with the goal of improving model fit. As shown in Table 4.4, wordings for item pair 17 and 18 as well as for item pair 41 and 48 are similar. To account for possible local dependence between these similarly worded pairs, I modeled each pair using an additional orthogonal "doublet" factor that was only measured by its corresponding two items and whose loadings were constrained to be equal to ensure model identification. The resulting seven-factor model (SFM) was fitted with 10 random starts using the same hyperparameters as the FFM.

The SFM loadings and factor correlation estimates are given in Tables 4.1 and 4.2, respectively, and are largely similar to the FFM estimates for the non-doublet factors. Fitting remained fast ( $M=188$ seconds, $S D=44$ seconds) and fairly stable (relative to the seven-factor reference model, loadings RMSE $M=0.038, S D=0.016$; intercepts RMSE $M=0.051, S D=0.021$; and factor 
correlation RMSE $M=0.032, S D=0.010$ ).

C2ST results for the SFM in Table 4.3 suggest that overall fit improved marginally relative to the FFM. In particular, mean test set accuracy for both KNNs and NNs was slightly lower for the SFM, suggesting that the SFM fit the data slightly better. However, all exact and approximate C2STs again rejected $H_{0}$ at $\alpha=0.05(\hat{p}<0.0001)$, suggesting that the SFM also failed to fit the data "well enough". The SFM's fit relative to the zero-factor baseline also improved only marginally: mean KNN- and NN-based C2ST-RFIs were slighly higher for the SFM than for the FFM, although these values remained far from the provisory cutoff of 0.9. Computation for all tests and fit indices remained fast.

Although the SFM appeared to improve overall fit only marginally relative to the FFM, piecewise fit appeared to improve more substantially. This improvement is evident in the SFM's PIs, which are shown in Figure 4.1b. In particular, NN-based PIs for the flagged item pairs are drastically lower for the SFM than for the FFM. Improvements in piece-wise fit for these item pairs were also observed for KNNs, although these improvements were relatively small since KNN-based PIs were much smaller than NN-based PIs for both the FFM and the SFM.

\subsection{Simulation Studies}

\subsubsection{Evaluating I-WAVE}

I investigate confirmatory I-WAVE's parameter recovery and computational efficiency as the number of IW samples $R$ increases and the log-likelihood approximation improves. The data generating model has $P=5$ factors and $J=505$-category items. Generating parameters are rounded estimates from the five-factor reference model in Section 4.2.1. I consider $R=1,5$, and 25 as well as $N=500,2500,12500$, and 62500 , resulting in 12 total simulation settings for each $R$ and $N$ combination. I conduct 100 replications at each setting. All analyses reused the optimization and inference model hyperparameters from Section 4.2.

Parameter recovery was assessed by computing the bias for each parameter as the mean deviation of the estimated parameter from the data generating parameter across replications, that is, $\operatorname{bias}(\hat{\xi}, \xi)=100^{-1} \sum_{a=1}^{100}\left[\hat{\xi}^{(a)}-\xi\right]$ where $\hat{\xi}^{(a)}$ is the estimated parameter at replication $a$ and $\xi$ 
is the data generating parameter. I also computed mean squared error (MSE) for each parameter as $\operatorname{MSE}(\hat{\xi}, \xi)=100^{-1} \sum_{a=1}^{100}\left[\hat{\xi}^{(a)}-\xi\right]^{2}$. Boxplots of parameter biases and MSEs for each simulation setting are displayed in Figures 4.2 and 4.3, respectively, with separate plots for factor loadings, factor correlations, and intercepts. All estimates become more accurate as the sample size increases. Loadings and factor correlations are highly biased for $R=1$ but appear nearly unbiased for $R \geq 5$. MSE tends to decrease with increasing $R$ for each $N$ setting. I note that several replications initially converged to poor local maxima when $N=500$ and $R \geq 5$ (specifically, 7 replications for $R=5$ and 10 replications for $R=25$ ). When $N \geq 2500$ and $R \geq 5$, poor local maxima occurred in one or fewer replications at each $(R, N)$ setting. Problematic replications were handled by refitting with a new random seed.

Line plots of fitting times for each simulation setting are displayed in Figure 4.4. Median fitting time decreases from around 116 seconds to around 96 seconds as $R$ increases from 1 to 5 , then increases to around 165 seconds as $R$ increases from 5 to 25 . For fixed $R$, median fitting time tends

to decrease slightly as $N$ increases. These results demonstrate that I-WAVE is computationally efficient even when the sample size is very large.

\subsubsection{Comparing I-WAVE to MH-RM}

In this study, I compare I-WAVE to the MML estimator implemented via MH-RM in a setting where the number of factors is large. I use the MH-RM implementation from the $\mathrm{R}$ package mirt, which has core functions written in both $\mathrm{R}$ and $\mathrm{C}++$ (Version 1.32.1 Chalmers 2012). The data generating model has $P=10$ factors measured by $J=100$ 5-category items. Generating parameters are again rounded estimates from the five-factor reference model in Section 4.2.1 with the parameters for items 51-100 set equal to the parameters for items 1-50. The factor correlation matrix is a $10 \times 10$ block diagonal matrix with rounded FFM estimates on the main-diagonals and zeros on the off-diagonals. I conduct 100 replications for each $N=1000,2500,5000$, and 10000 . For I-WAVE, I set $R=5$ and set the learning rate optimization hyperparameter to 0.0025 to ensure the IW-ELBO does not diverge due to the large number of factors (for details, see Urban \& Bauer 2021). I-WAVE converged to poor local maxima once each when $N=1000$ and 2500 , both of 

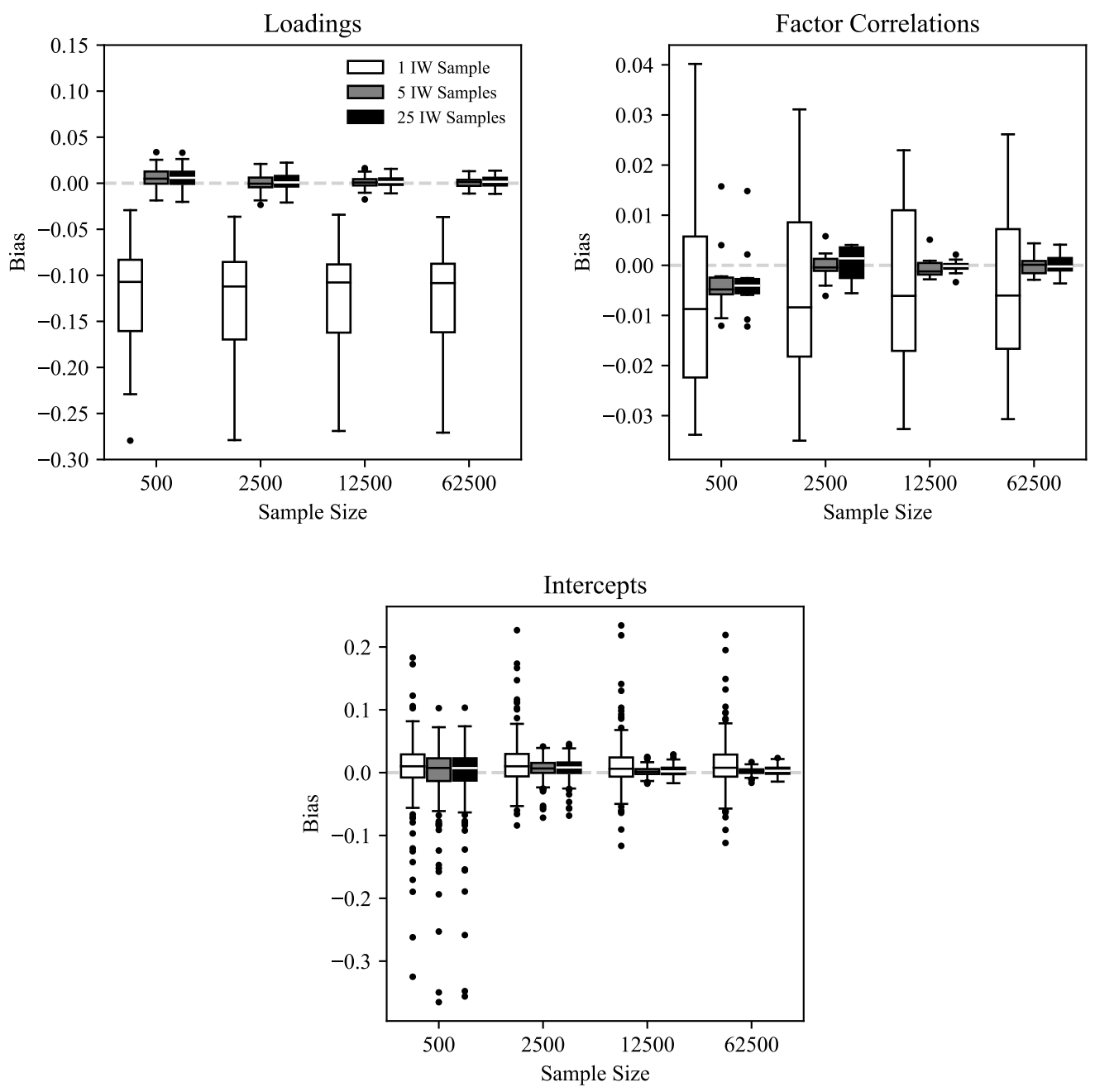

Figure 4.2: Parameter bias for the importance-weighted amortized variational estimator (I-WAVE). Three settings for the number of importance-weighted (IW) samples are compared. 

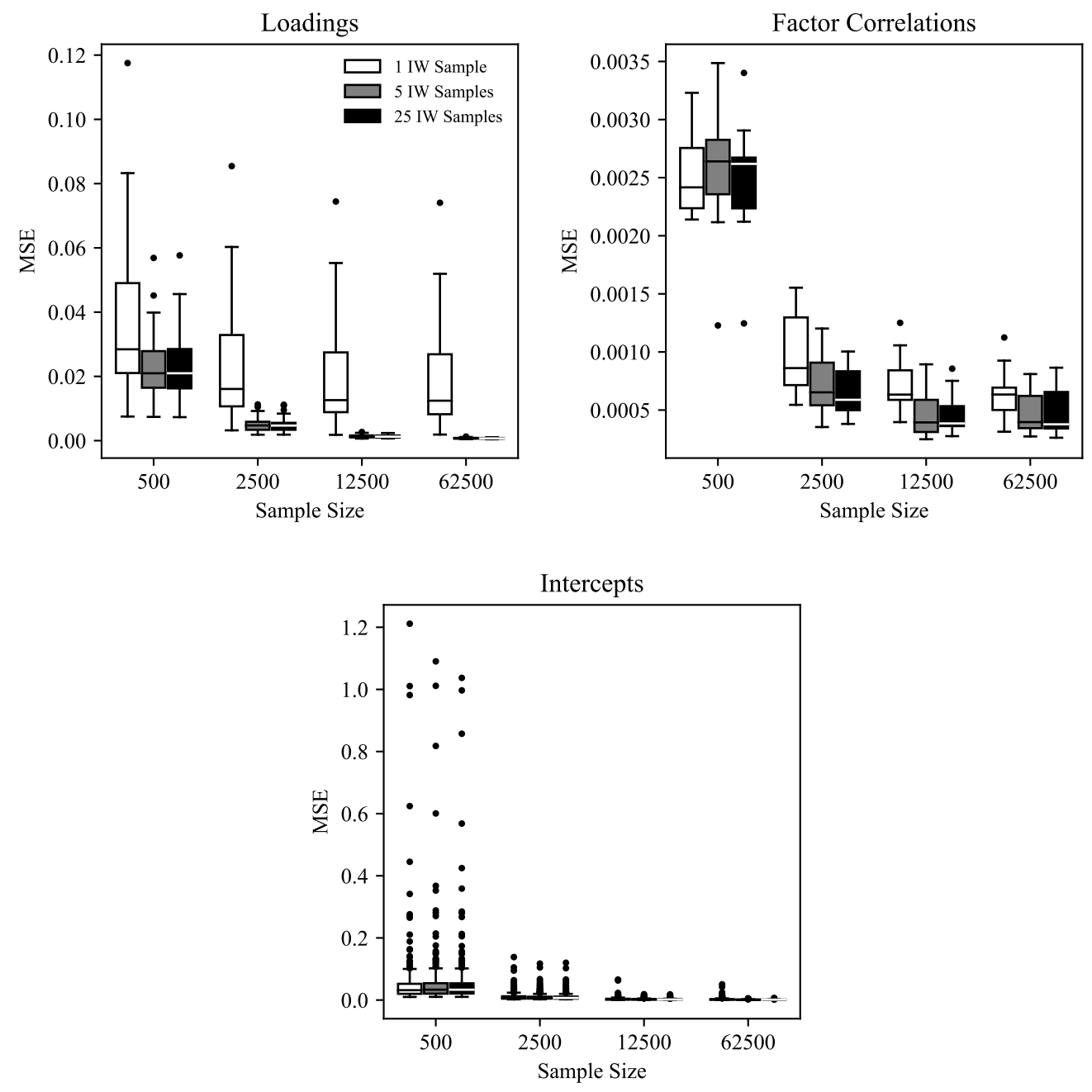

Figure 4.3: Parameter mean squared error (MSE) for I-WAVE. 


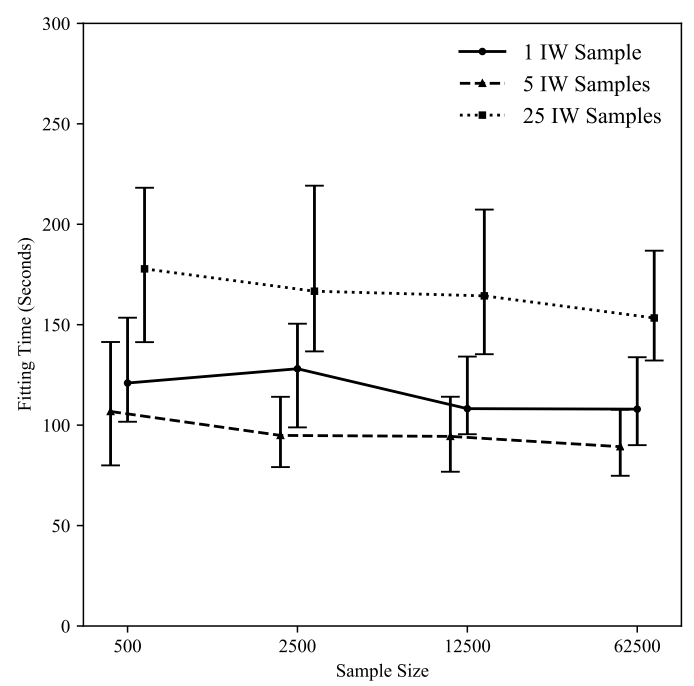

Figure 4.4: Fitting times for I-WAVE. Markers indicate medians, while error bars indicate $25 \%$ and $75 \%$ quantiles.

which were handled by refitting with a new random seed. I set MH-RM hyperparameters to the mirt package defaults, which performed well across $N$ settings.

Simulation results are presented in Figures 4.5 and 4.6. Both methods obtain comparable estimates in all $N$ settings and have MSE decreasing toward zero with increasing $N$. I-WAVE may obtain slightly better estimates than MH-RM for $N=1000$, and vice versa for $N=10000$, although the differences appear to be somewhat negligible. Parameter biases for both methods were also comparable and are not shown. I-WAVE is faster than MH-RM in all settings: the median fitting time for I-WAVE stays around three minutes and decreases slightly as $N$ increases, whereas the median fitting time for MH-RM is around 4.5 minutes when $N=1000$ and increases to around 18 minutes when $N=10000$. I note that the MH-RM implementation in the commercially available flexMIRT software (Cai 2017) has core functions written in C++ and is likely faster than mirt, although even this implementation would become slower as $N$ increases. Additionally, I-WAVE could achieve a potentially large speedup using a GPU, whereas a similar speedup is not currently available for MH-RM.

\subsubsection{Evaluating Approximate C2STs in a Non-IFA Setting}

I conduct a small simulation study to verify that $\mathrm{KNN}$ - and $\mathrm{NN}$-based approximate C2STs have empirical type I error close to $\alpha=0.05$ as well as empirical power close to values predicted by the 

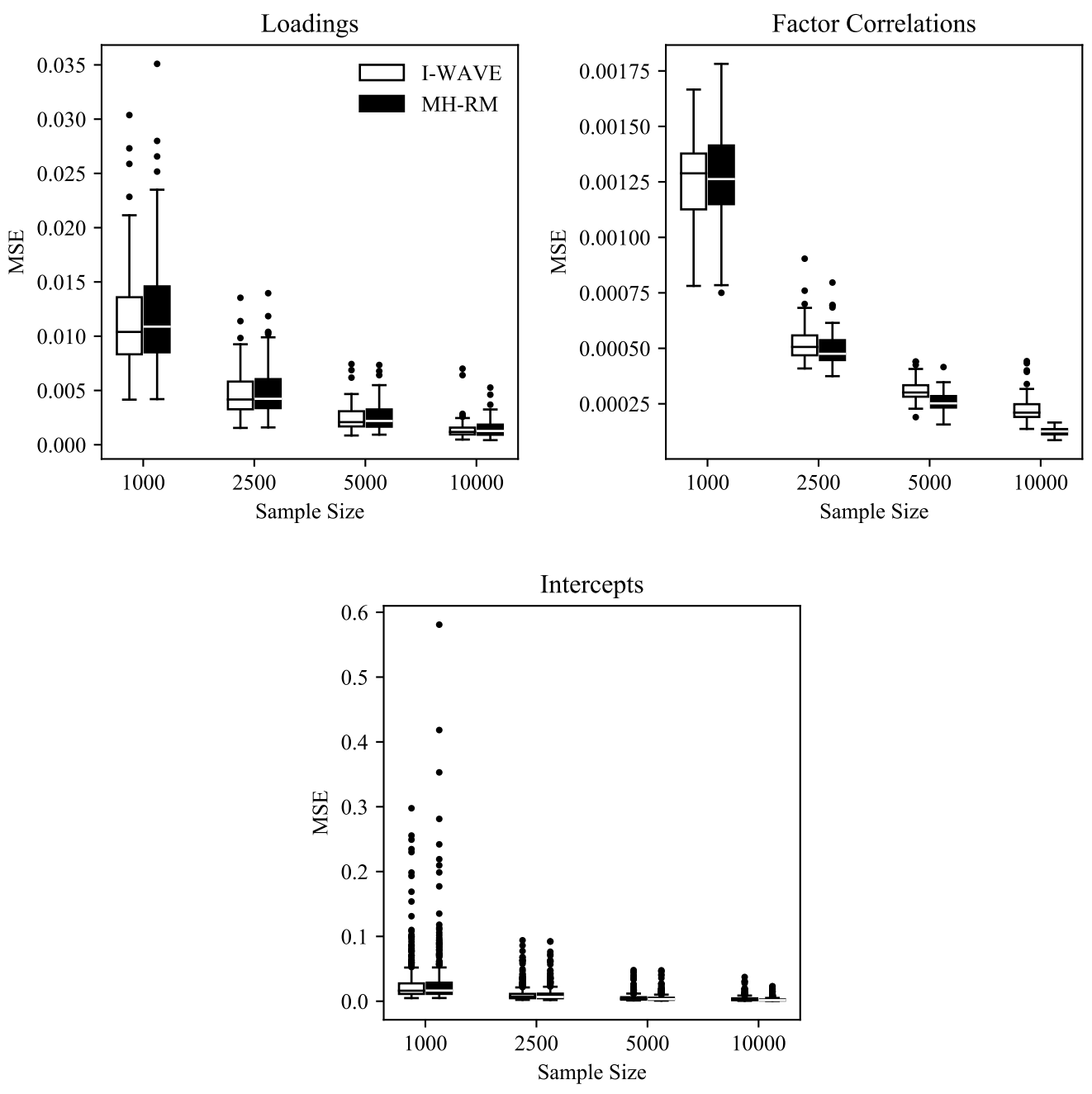

Figure 4.5: MSE for I-WAVE and the marginal maximum likelihood estimator. $\mathrm{MH}-\mathrm{RM}=$ Metropolis-Hastings Robbins-Monro. 


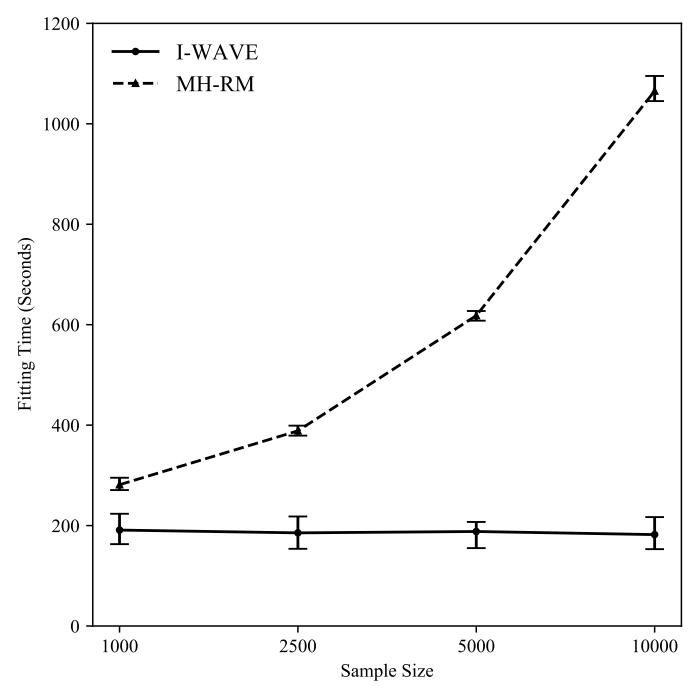

Figure 4.6: Fitting times for I-WAVE and the marginal maximum likelihood estimator.

formula in Theorem 1. Type I error rates for exact C2STs were empirically verified by Lopez-Paz and Oquab (2017) and are not considered here. Since it is difficult to control the effect size $\varepsilon$ in the IFA setting, I instead consider real-valued data drawn from tractable distributions whereby $\varepsilon$ is precisely controlled. The use of real-valued data requires that KNNs be modified to use the Euclidean distance metric $d\left(\mathbf{u}_{i}, \mathbf{u}_{i^{\prime}}\right)=\left\|\mathbf{u}_{i}-\mathbf{u}_{i^{\prime}}\right\|_{2}$ rather than the Hamming distance metric.

I begin with the type I error experiments. For each replication $a=1, \ldots, 100$, I simulate data by drawing two samples $\left\{x_{i}^{(a)}\right\}_{i=1}^{N} \sim \mathbb{P}=\operatorname{Uniform}\left(x_{i} \mid 0,1\right)$ and $\left\{y_{i}^{(a)}\right\}_{i=1}^{N} \sim \hat{\mathbb{P}}=\operatorname{Uniform}\left(y_{i} \mid\right.$ $0.05,1.05)$ where $N=250,500,1000,2500,5000$, and 10000. $\mathbb{P}$ and $\hat{\mathbb{P}}$ are shown in Figure 4.7. Close to $95 \%$ of the observations in each data set will fall in the region where $\mathbb{P}$ and $\hat{\mathbb{P}}$ overlap (i.e., in $[0.1,1]$ ) and will be indistinguishable since $\mathbb{P}=\hat{\mathbb{P}}$. On the other hand, close to $5 \%$ of the observations will fall either to the left or the right of the overlapping region (i.e., either in $[0,0.1)$ or $(1,1.05]$, respectively) and will be perfectly distinguishable since $\hat{\mathbb{P}}=0$ to the left and $\mathbb{P}=0$ to the right. This implies that the maximum obtainable test set classification accuracy is close to 0.525. I therefore test $H_{0}: a c c=0.525$ against $H_{1}: a c c>0.525$ so that the effect size $\varepsilon=0$. Figure 4.8 shows that both $\mathrm{KNN}$ - and NN-based approximate C2STs have rejection rates close to the nominal level in most $N$ settings and test set classification accuracies converging to 0.525 as $N_{\text {test }}$ increases. NN-based approximate C2STs have low type I error rates when $N_{\text {test }} \leq 500$, likely 


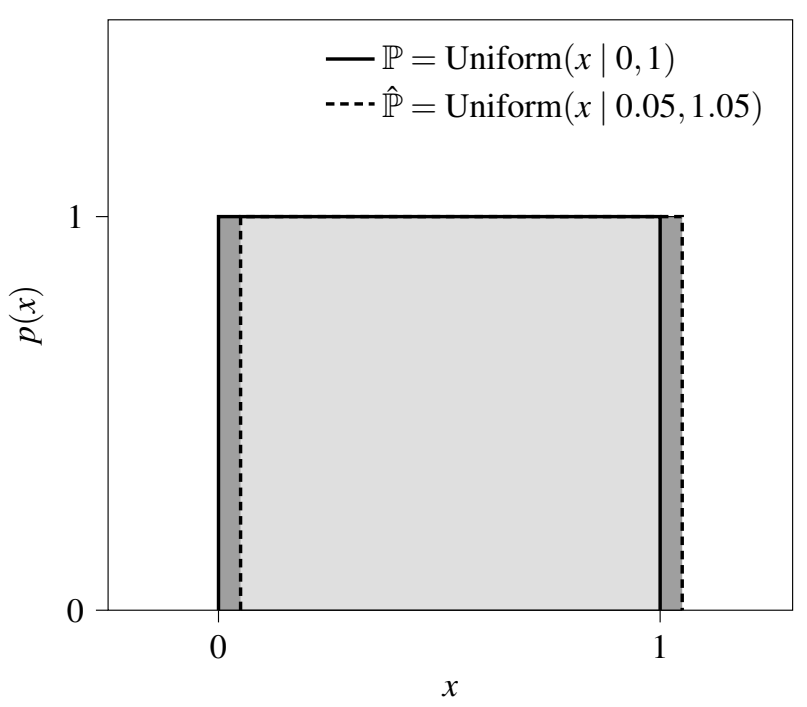

Figure 4.7: Uniform generating distributions used to verify the type I error rate for approximate C2STs. Observations falling in the overlapping (light gray) region are indistinguishable, while observations falling in the non-overlapping (dark gray) regions are perfectly distinguishable.

because the NNs overfitted the training data in these small $N$ settings.

To assess empirical power, I follow the same procedure as above except I now draw $\left\{y_{i}^{(a)}\right\}_{i=1}^{N} \sim$ $\widehat{\mathbb{P}}=$ Uniform $\left(y_{i} \mid 0.1,1.1\right)$. By similar reasoning to that given above, the maximum obtainable test set classification accuracy in this setting is close to 0.55 . I again test $H_{0}: a c c=0.525$ against $H_{1}:$ acc $>0.525$ so that now the effect size $\varepsilon=0.025$. Figure 4.9 shows that all approximate C2STs have both power converging to one and test set classification accuracies converging to 0.55 as $N_{\text {test }}$ increases. NNs do not have very low power when $N_{\text {test }} \leq 500$ despite having low type I error rates in these settings. Power values predicted by the formula in Theorem 1 are close to the empirical power values for all tests.

\subsubsection{Evaluating C2STs and C2ST-RFIs in a Confirmatory IFA Setting}

I now investigate the proposed GOF assessment methods' performance in settings where the fitted IFA model is correctly or incorrectly specified. I consider two data generating models for $J=50$ five-category items: (1) the same FFM considered in Section 4.3.1 as well as (2) an SFM with five correlated factors each measured by 10 items, one orthogonal doublet factor measured by items 17 and 18, and another orthogonal doublet factor measured by items 41 and 48 . Generating parameters for (2) are rounded estimates from the seven-factor reference model in Section 4.2.2. 

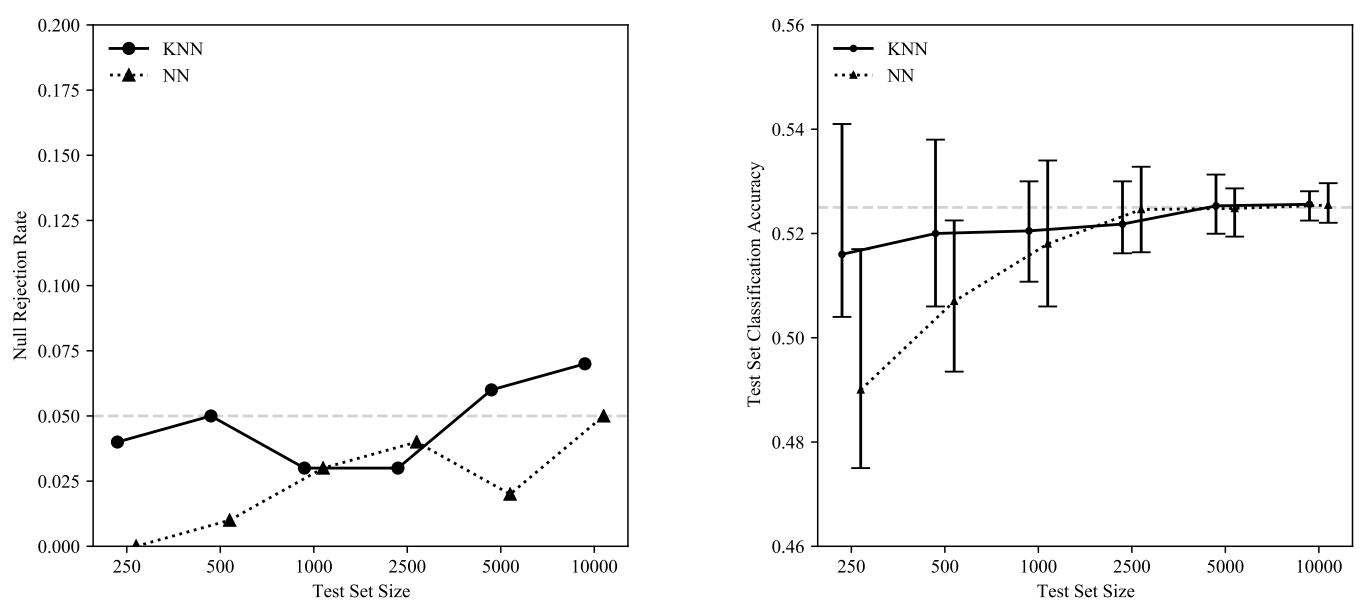

Figure 4.8: Empirical type I error and test set classification accuracy for KNN- and NN-based approximate C2STs.
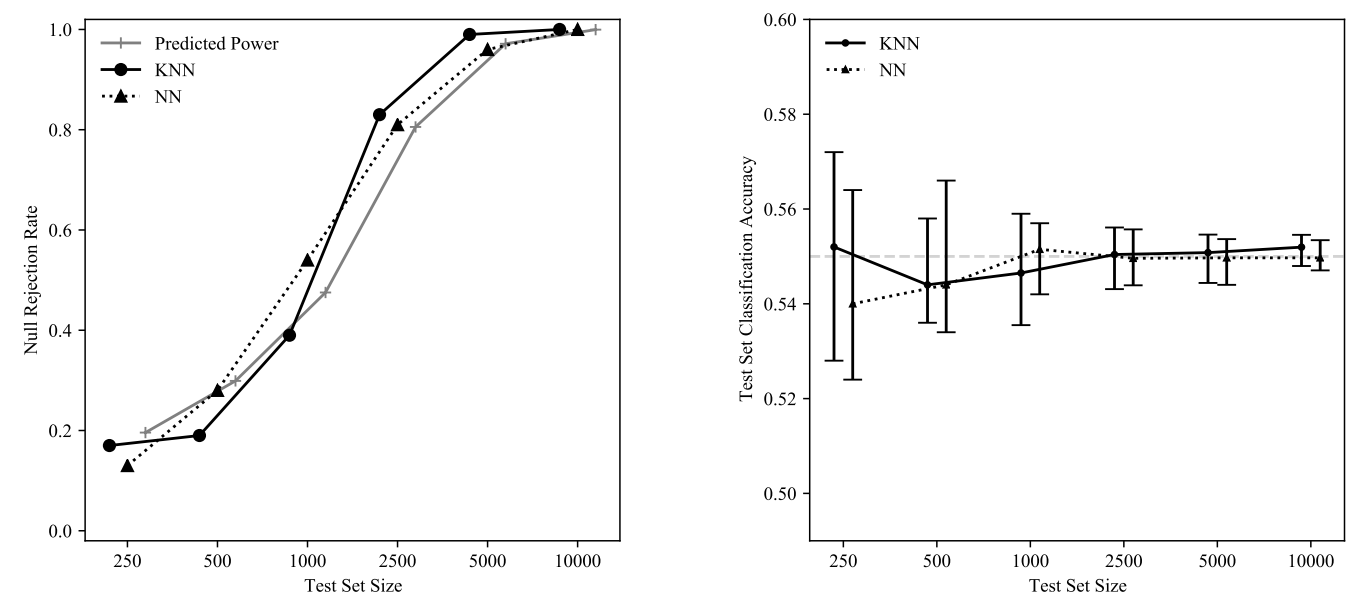

Figure 4.9: Empirical power and and test set classification accuracy for KNN- and NN-based approximate C2STs. 
I simulate 100 data sets from (1) and (2) for each $N=750,1250,2500,5000$, and 10000 . Using I-WAVE with the same hyperparameters as in Section 4.2, I fit two models to each data set: (a) an FFM with the same specification as (1) and (b) an SFM with the same specification as (2). This leads to four different settings for each combination of data generating model and fitted model. Viable GOF assessment methods should indicate near perfect fit for settings (1a) and (2b), which have correctly specified fitted models, as well as for setting (1b), which has an overspecified fitted model. Setting (2a), on the other hand, has an underspecified fitted model and should demonstrate poorer fit as $N$ increases. Although it might be expected that $a c c=0.5$ in settings with correctly specified and overspecified models, it is also feasible that IFA model parameter estimate uncertainty leads to acc slightly greater than 0.5 . To investigate both possibilities, I assess GOF for all simulation settings using KNN- and NN-based exact C2STs where $\delta=0$ as well as approximate C2STs where $\delta=0.025$, which respectively correspond to $H_{0}:$ acc $=0.5$ and $H_{0}: a c c=0.525$.

Rejection rates at signficance level $\alpha=0.05$ as well as test set classification accuracies for the overspecified SFM in setting (1b) are shown in Figure 4.10a. Results for the correctly specified models in settings (1a) and (2b) were nearly identical and are not shown. Rejection rates remain well below the nominal level and test set classification accuracies come close to 0.5 as $N_{\text {test }}$ increases, suggesting that the SFM fits the simulated data nearly perfectly. The near-zero rejection rates likely occurred because there was almost no signal in the training data, leading classifiers to overfit to noise and perform worse than random chance for small to moderate $N$. A similar phenomenon was observed in Sect. 4.3.3, where it appeared to have a negligible impact on empirical power.

C2ST results for the underspecified fitted model in setting (2a) are shown in Figure 4.10b. NN-based C2STs performed well, with classification accuracies exceeding 0.5 and rejection rates tending to one as $N_{\text {test }}$ increases. KNN-based C2STs, however, performed relatively poorly, with classification accuracies only marginally exceeding 0.5 , exact tests only obtaining moderate rejection rates when $N_{\text {test }}=10000$, and approximate tests always failing to reject $H_{0}$. These results 

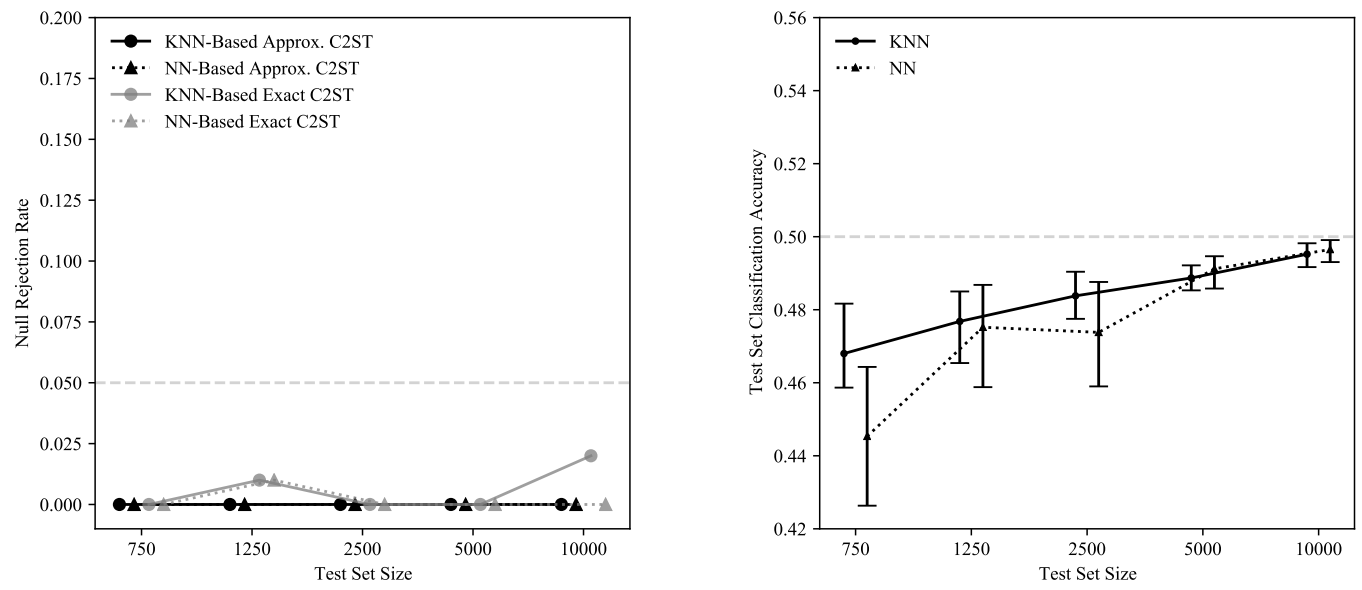

(a) An overspecified seven-factor fitted model.
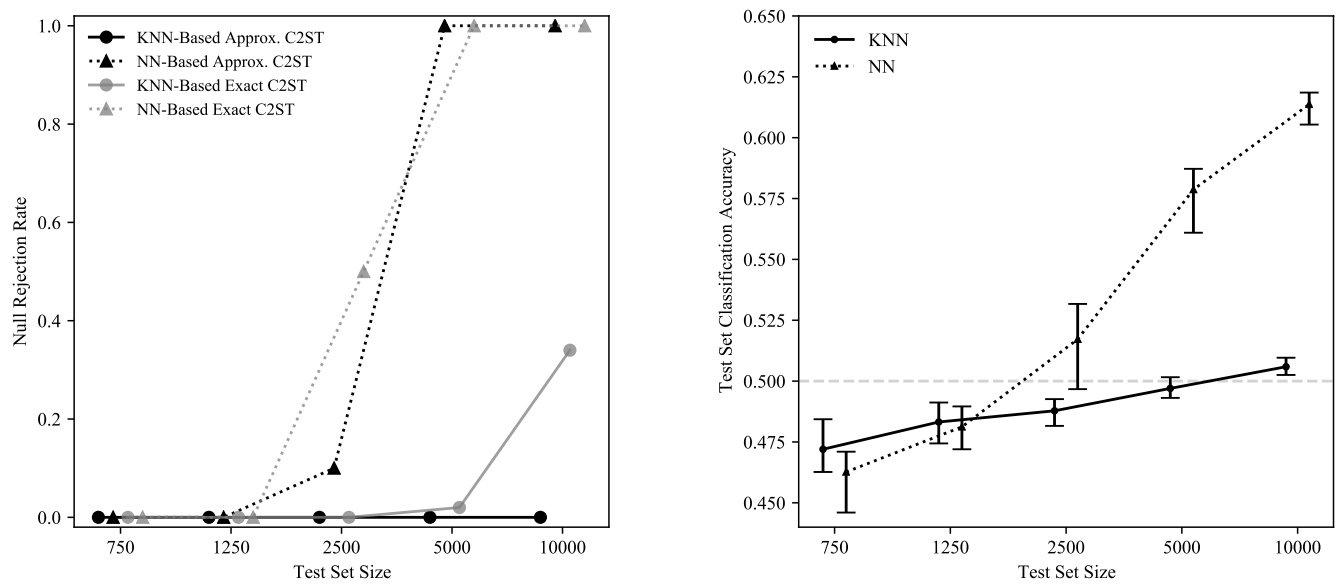

(b) An underspecified five-factor fitted model.

Figure 4.10: Rejection rates as well as test set classification accuracies for approximate and exact C2STs. 

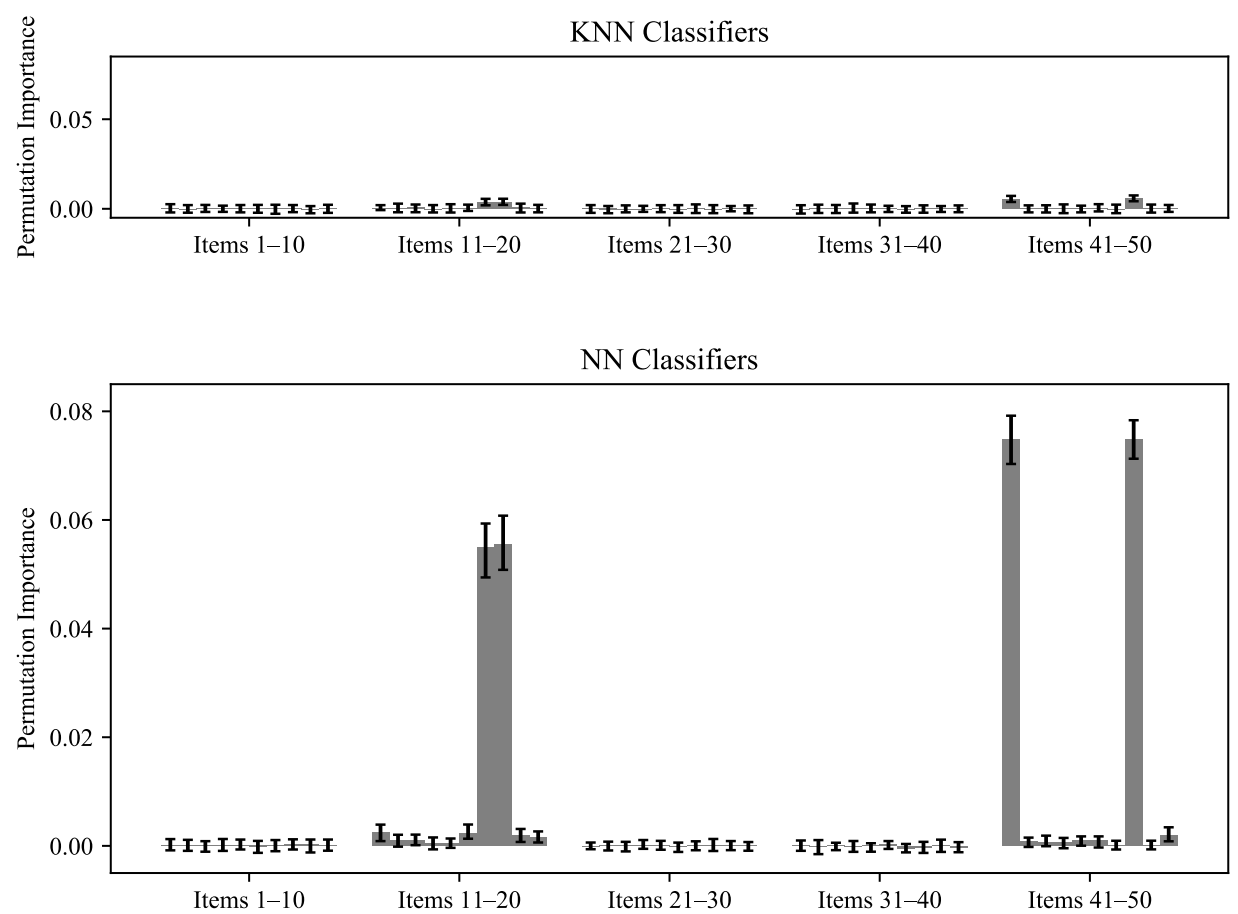

Figure 4.11: Permutation importances for the underspecified five-factor fitted model when $N=$ 10000 .

mirror NNs' superior performance in Section 4.2. I also demonstrate the viability of PIs by assessing the underspecified fitted model's item-level fit when $N=10000$. Results in Figure 4.11 show that NNs clearly flagged items $17,18,41$, and 48 as poor fitting with all other items having PIs near zero. KNNs also flagged these items, although PIs were much smaller than for NNs.

Boxplots of C2ST-RFIs for the overspecified SFM in setting (1b) as well as for the underspecified FFM in setting (2a) are shown in Figures 4.12a and 4.12b, respectively. C2ST-RFIs for the correctly specified models in settings (1a) and (2b) were nearly identical to C2ST-RFIs for (1b) and are not shown. The means of the sampling distributions of both KNN- and NN-based C2STRFIs appear to depend on $N$. C2ST-RFIs for both (1b) and (2a) start out larger than one when $N_{\text {test }}=750$, with RFIs for (1b) tending to one and RFIs for (2a) tending to values smaller than one as $N_{\text {test }}$ increases. These results suggest that the SFM fit its data almost perfectly and that the FFM fit its data relatively poorly, although classifiers overfitted to noise in the training data for 


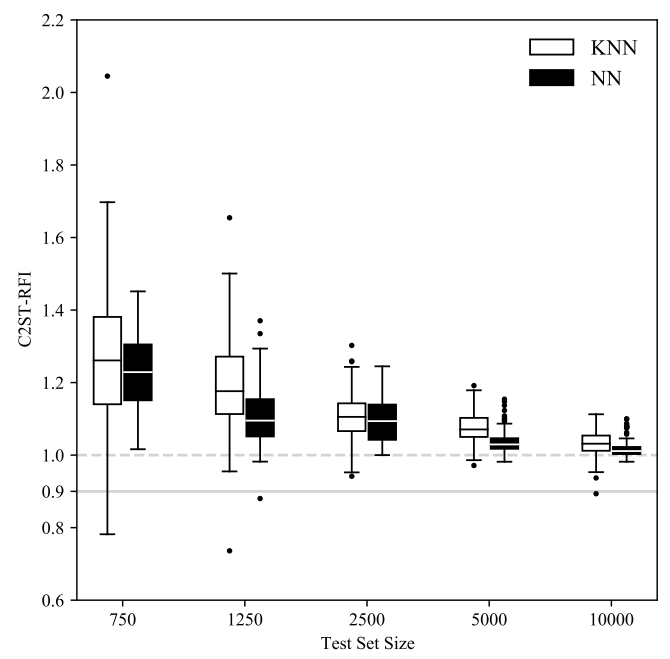

(a) An overspecified seven-factor fitted model.

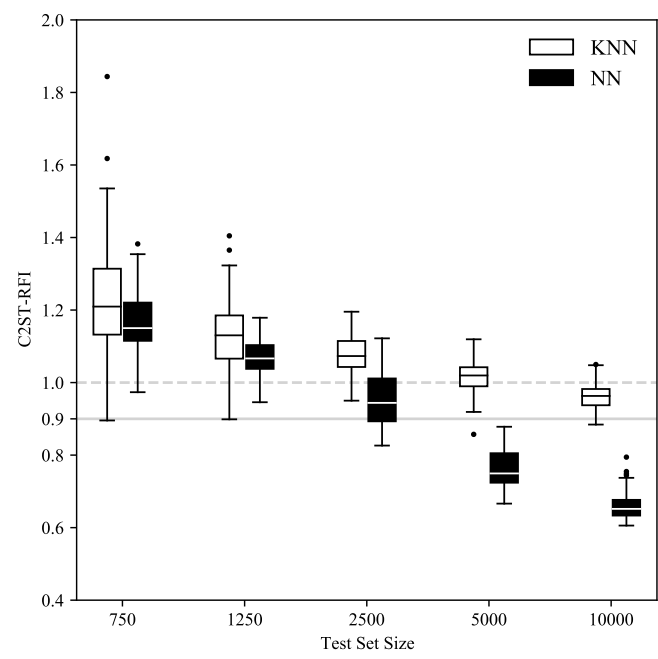

(b) An underspecified five-factor fitted model.

Figure 4.12: C2ST-RFIs for simulated data. The provisory cutoff of 0.9 is marked with a solid line.

small $N_{\text {train. }}$. The provisory cutoff of 0.9 obtains similar rejection rates to those obtained by C2STs: essentially no C2ST-RFIs fall below the threshold in setting (1a) (i.e., the rejection rate remains near zero), all NN-based C2ST-RFIs in setting (2b) fall below the threshold as $N_{\text {test }}$ increases (i.e., the rejection rate tends to one), and $\mathrm{KNN}$-based C2ST-RFIs in setting (2b) start falling below the threshold for large $N_{\text {test }}$ (i.e., the rejection rate slowly increases).

I assessed each GOF assessment method's computational efficiency by computing the total time required to sample all synthetic data and to fit (and possibly tune hyperparameters for) all associated classifiers. Total run times for C2STs and for C2ST-RFIs computed in simulation setting (2a) are shown in Figures 4.13a and 4.13b, respectively. Run times for other simulation settings were very similar and are not shown. Run times increase with increasing $N_{\text {test }}$, with KNNs always running faster than NNs. For instance, when $N_{\text {test }}=10000, \mathrm{KNN}$-based C2STs and C2ST-RFIs have median run times around 4 and 9 seconds, respectively, whereas their NN-based counterparts have median run times around 18 and 40 seconds. This finding is likely attributable to the fact that no KNN hyperparameters are tuned during fitting as well as to the fact that $N$ is never extremely large (e.g., $N \geq 100000$ ), in which case NNs would potentially be much faster than KNNs. Additionally, absolute run times are never prohibitively large, suggesting that the better-performing 


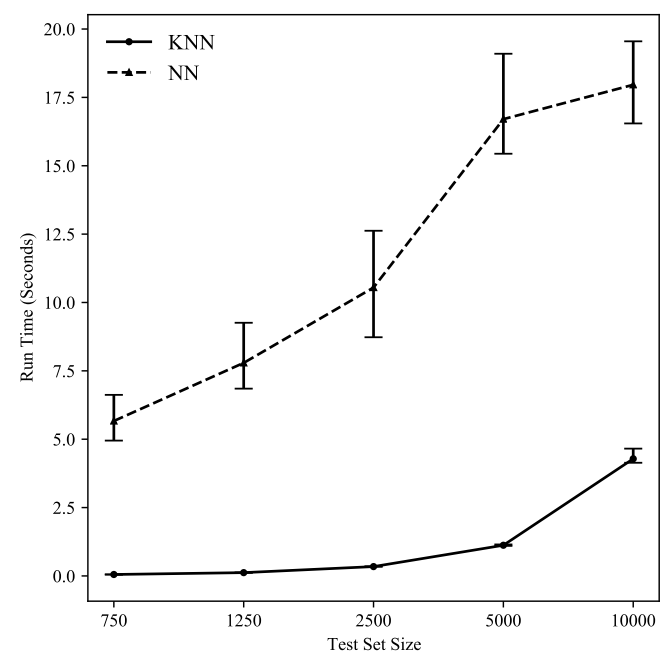

(a) C2ST run times.

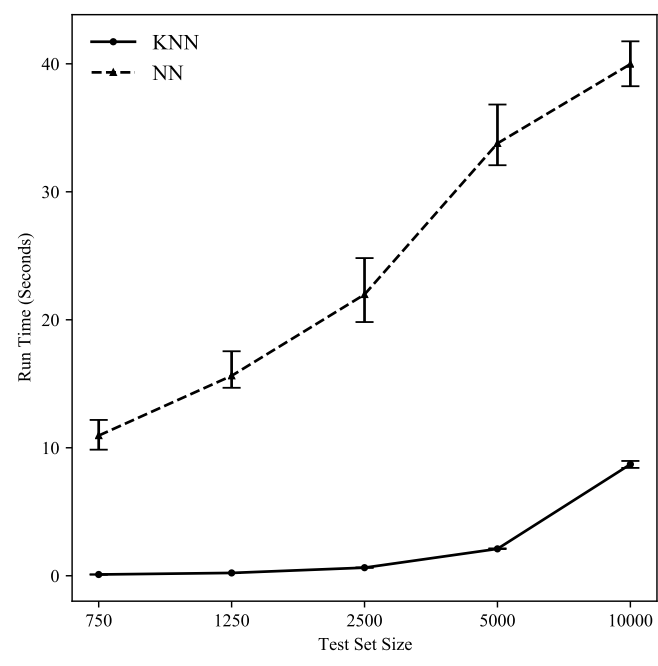

(b) C2ST-RFI run times.

Figure 4.13: Run times for KNN- and NN-based C2STs and C2ST-RFIs from the simulation setting with the underspecified five-factor model.

NNs are still a viable choice in practice. 


\section{DISCUSSION}

This thesis is concerned with the theoretical properties and empirical performance of machine learning-based parameter estimation and goodness-of-fit assessment methods for large-scale confirmatory item factor analysis. An importance-weighted amortized variational estimator implemented via a deep learning algorithm demonstrated improved parameter recovery as the sample size increased and obtained comparable estimates to those obtained by the MML estimator implemented via the MH-RM algorithm. The deep learning algorithm's computational efficiency appears to be essentially independent of the sample size, enabling fitting even with extremely large samples. A simulation-based test of exact fit called the classifier two-sample test was described and extended into a test of approximate fit as well a relative fit index. Approximate C2STs and C2ST-RFIs successfully identified when an IFA model was correctly or incorrectly specified. C2ST results suggested that more flexible (but potentially harder to tune) classifiers such as NNs performed better than less flexible (but potentially easier to tune) classifiers such as KNNs. A permutation importance technique was demonstrated as a tool for exploring piece-wise model fit.

The proposed methods have a number of limitations and extensions that may be addressed in future work.

First, optimization for I-WAVE may be improved to ensure both (1) stabler and (2) faster convergence. Regarding (1), I note that I-WAVE occasionally converged to poor local maxima, particularly with small sample sizes. I handled this issue by refitting the model with new random starting values. I also manually reduced the learning rate optimization hyperparameter when the latent dimension was large to ensure stable convergence. It may be feasible to mitigate insta-

bility using more computationally intensive procedures for choosing starting values such as Cai's (2010b) stochastic EM procedure or Y. Chen, Li, and Zhang's (2019) singular value decomposition 
procedure. In addition, the automatic procedures for choosing starting values and for tuning optimization hyperparameters recommended by Agrawal, Sheldon, and Domke (2020) in the context of general amortized importance-weighted VI may help reduce user burden. At present, I recommend trying multiple random starts to mitigate convergence to poor local maxima. I note that this is typically considered good practice for IFA models fitted via most popular estimators with nonconvex objective functions (e.g., the MML estimator) since convergence to the global maximum is not guaranteed in general. Regarding (2), I note that convergence could potentially be sped up using a variant of natural gradient descent (e.g., Hoffman, Blei, Wang, \& Paisley 2013; Tang \& Ranganath 2019). This would be similar in spirit to recent work by Liu (2020) in which computational efficiency for a JML estimator was enhanced via a Riemannian optimization procedure that exploits the manifold structure of the parameter space.

Second, computing standard errors (SEs) and handling missing data with I-WAVE were not discussed. Approximate SEs may be obtained by evaluating the observed information matrix at the parameter estimates obtained by maximizing the IW-ELBO, then inverting this matrix blockwise (Hui et al. 2017). As noted by Urban and Bauer (2021), SEs will likely be small for the large-scale applications considered here. Procedures for handling missing-at-random data (Mattei \& Frellsen 2018) as well as missing-not-at-random data (Ipsen, Mattei, \& Frellsen 2021) have been developed for general amortized importance-weighted VI and can be straightforwardly utilized for I-WAVE.

Third, using approximate C2STs to assess person and piece-wise fit may be more thoroughly investigated. In terms of person fit, the approach suggested in Sect. 3.1 remains to be investigated via simulation studies. In terms of piece-wise fit, the permutation importance measures applied here have shortcomings including (1) unknown sampling distributions and (2) degraded performance when predictors are highly correlated (e.g., Hooker \& Mentch 2019). For (1), future work may explore whether accurate $p$-values and confidence intervals for estimated PIs can be obtained via parametric approximations (Altmann, Toloşi, Sander, \& Lengauer 2010) or bootstrap resampling. For (2), it would likely be fruitful to explore alternative approaches based on Shapley 
additive explanations (SHAP; Lundberg \& Lee 2017). SHAP is a game theoretic method for explaining fitted classifiers' predictions and has several extensions that help prevent its performance from degrading in the presence of multicollinearity (Aas, Jullum, \& Løland 2021; Basu \& Maji 2020; Sellereite \& Jullum 2020). I note, however, that SHAP is less computationally efficient than PI, potentially hampering its application to very large-scale data.

Fourth, C2ST-RFIs may be improved by developing better (1) cutoff criteria, (2) baseline models, and (3) model complexity penalties. For (1), I studied the performance of C2ST-RFIs under a fairly narrow range of modeling conditions. Although my provisory cutoff criterion of 0.9 performed well in these conditions, further research is needed to develop more robust cutoff criteria for evaluating model fit in practice. For (2), I focused on zero-factor or "independence" baseline models to closely parallel how fit is assessed in the SEM literature. However, Maydeu-Olivares (2013b) notes that zero-factor baseline models may be overly unrealistic in that they disregard inter-item correlations, potentially inflating our sense of the proposed model's relative value. A more realistic alternative is suggested by Urban and Bauer's (2021, Section 7.4) note that I-WAVE can be used to fit a fully nonlinear factor model in which the inverse link function between the observed and latent variables is approximated using an $\mathrm{NN}$ : first fit the proposed model as well as the fully nonlinear baseline model, then construct a "badness-of-fit" index that measures the proportional decrement in fit obtained by moving from the highly flexible baseline to the less flexible model of interest (e.g., using the ratio $\widehat{a c c}_{\text {base }} / \widehat{a c c}_{\text {prop }}$ ). For (3), I employed a complexity penalty based on the number of fitted parameters. Although this parameter counting approach is fast, alternative penalties that take into account IFA models' functional forms would provide more accurate characterizations of complexity that could be used to compare different models with the same number of parameters (e.g., Bonifay \& Cai 2017).

In summary, the methods considered in this thesis may provide feasible and promising frameworks for testing hypotheses about the latent structure underlying large-scale item response data in a computationally efficient manner. Both I-WAVE and C2STs are highly flexible frameworks that may be extended in a variety of ways. Some of these extensions are discussed above, some 
are discussed in the vast machine learning literature, and some are yet to be conceived. I view this work as part of a dialogue between machine learning and psychometrics that is leading to the development of new extensions and applications with the potential to positively impact both fields. 


\section{REFERENCES}

Aas, K., Jullum, M., \& Løland, A. (2021). Explaining individual predictions when features are dependent: More accurate approximations to Shapley values. Artificial Intelligence, 298, 1-28. doi: 10.1016/j.artint.2021.103502

Agrawal, A., Sheldon, D., \& Domke, J. (2020). Advances in black-box VI: Normalizing flows, importance weighting, and optimization. 34th Conference on Advances in Neural Information Processing Systems (NeurIPS 2020). Retrieved from http://arxiv.org/abs/ 2006.10343

Altmann, A., Toloşi, L., Sander, O., \& Lengauer, T. (2010). Permutation importance: A corrected feature importance measure. Bioinformatics, 26(10), 1340-1347. doi: 10.1093/ bioinformatics/btq134

Anderson, T. W., \& Rubin, H. (1956). Statistical inference in factor analysis. Proceedings of the Third Berkeley Symposium on Mathematical Statistics and Probability, V, 111.

Basu, I., \& Maji, S. (2020). Multicollinearity correction and combined feature effect in Shapley values. Retrieved from http://arxiv.org/abs/2011.01661

Béguin, A. A., \& Glas, C. A. (2001). MCMC estimation and some model-fit analysis of multidimensional IRT models. Psychometrika, 66(4), 541-562. doi: 10.1007/BF02296195

Bentler, P. M. (1990). Comparative fit indices in structural equation models. Psychological Bulletin, 107(2), 238-246.

Bentler, P. M. (1995). EQS structural equations program manual. Encino, CA.

Bentler, P. M., \& Bonett, D. G. (1980). Significance tests and goodness of fit in the analysis of covariance structures. Psychological Bulletin, 88(3), 588-606. doi: 10.1037/0033-2909.88 .3 .588

Biernacki, C., Celeux, G., \& Govaert, G. (2003). Choosing starting values for the EM algorithm for getting the highest likehood in multivariate Gaussian mixture models. Computational Statistics and Data Analysis, 41(3-4), 561-575. doi: 10.1016/S0167-9473(02)00163-9

Blei, D. M., Kucukelbir, A., \& McAuliffe, J. D. (2017). Variational inference: A review for statisticians. Journal of the American Statistical Association, 112(518), 859-877. doi: 10 $.1080 / 01621459.2017 .1285773$

Bock, R. D., \& Aitkin, M. (1981). Marginal maximum likelihood estimation of item parameters: Application of an EM algorithm. Psychometrika, 46(4), 443-459. doi: 10.1007/ BF02293801

Bock, R. D., Gibbons, R., \& Muraki, E. (1988). Full-information item factor analysis. Applied 
Psychological Measurement, 12(3), 261-280. doi: 10.1177/014662168801200305

Bollen, K. A. (1989a). A new incremental fit index for general structural equation models. Sociological Methods \& Research, 17(3), 303-316.

Bollen, K. A. (1989b). Structural equations with latent variables. John Wiley \& Sons, Inc.

Bonifay, W., \& Cai, L. (2017). On the complexity of item response theory models. Multivariate Behavioral Research, 52(4), 465-484. Retrieved from https://doi.org/10.1080/ 00273171.2017 .1309262 doi: 10.1080/00273171.2017.1309262

Bottou, L., Curtis, F. E., \& Nocedal, J. (2018). Optimization methods for large-scale machine learning. SIAM Review, 60(2), 223-311. doi: 10.1137/16M1080173

Breiman, L. (2001). Random forests. Machine Learning, 45(1), 5-32. doi: 10.1007/ 9781441993267_5

Burda, Y., Grosse, R., \& Salakhutdinov, R. (2016). Importance weighted autoencoders. 4th International Conference on Learning Representations, ICLR 2016 - Conference Track Proceedings, 1-14.

Butcher, J. N., Dahlstrom, W., Graham, J., Tellegen, A., \& Kaemmer, B. (1989). MMPI-2: Manual for administration and scoring. Minneapolis, MN: University of Minnesota Press.

Cai, L. (2010a). High-dimensional exploratory item factor analysis by a Metropolis-Hastings Robbins-Monro algorithm. Psychometrika, 75(1), 33-57. doi: 10.1007/s11336-009-9136-x

Cai, L. (2010b). Metropolis-Hastings Robbins-Monro algorithm for confirmatory item factor analysis. Journal of Educational and Behavioral Statistics, 35(3), 307-335. doi: 10.3102/ 1076998609353115

Cai, L. (2017). flexMIRT®: Flexible multilevel multidimensional item analysis and test scoring. Chapel Hill, NC: Vector Psychometric Group.

Cai, L., \& Hansen, M. (2013). Limited-information goodness-of-fit testing of hierarchical item factor models. British Journal of Mathematical and Statistical Psychology, 66(2), 245-276. doi: 10.1111/j.2044-8317.2012.02050.x

Chalmers, R. P. (2012). Mirt: A multidimensional item response theory package for the R environment. Journal of Statistical Software, 48(April 2012). doi: 10.18637/jss.v048.i06

Chen, X., Liu, S., Sun, R., \& Hong, M. (2019). On the convergence of a class of ADAM-type algorithms for non-convex optimization. Internation Conference on Learning Representations.

Chen, Y., Filho, T. S., Prudêncio, R. B. C., Diethe, T., \& Flach, P. (2019). $\beta 3$-IRT : A new item response model and its applications. Proceedings of the 22nd International Conference on 
Artificial Intelligence and Statistics (AISTATS 2019), 89.

Chen, Y., Li, X., \& Zhang, S. (2019). Joint maximum likelihood estimation for high-dimensional exploratory item factor analysis. Psychometrika, 84(1), 124-146. doi: 10.1007/s11336-018 $-9646-5$

Cho, A. E., Wang, C., Zhang, X., \& Xu, G. (2020). Gaussian variational estimation for multidimensional item response theory. British Journal of Mathematical and Statistical Psychology. doi: 10.1111/bmsp.12219

Chwialkowski, K., Ramdas, A., Sejdinovic, D., \& Gretton, A. (2015). Fast two-sample testing with analytic representations of probability measures. Advances in Neural Information Processing Systems, 1981-1989.

Cremer, C., Li, X., \& Duvenaud, D. (2018). Inference suboptimality in variational autoencoders. 35th International Conference on Machine Learning, ICML 2018, 3, 1749-1760.

Cudeck, R., \& Henly, S. J. (1991). Model selection in covariance structures analysis and the "problem" of sample size: A clarification. Psychological Bulletin, 109(3), 512-519. doi: 10.1037/0033-2909.109.3.512

Curi, M., Converse, G. A., \& Hajewski, J. (2019). Interpretable variational autoencoders for cognitive models. In International joint conference on neural networks (pp. 1-8). Budapest, Hungary. doi: 10.1109/IJCNN.2019.8852333

Cybenko, G. (1989). Approximation by superpositions of a sigmoidal function. Mathematics of Control, Signals, and Systems, 2, 303-314.

Domke, J., \& Sheldon, D. (2018). Importance weighting and variational inference. Advances in Neural Information Processing Systems, 2018-Decem, 4470-4479.

Duda, R. O., Hart, P. E., \& Stork, D. G. (2001). Pattern classification. New York, NY: Wiley.

Edwards, M. C. (2010). A Markov chain Monte Carlo approach to confirmatory item factor analysis. Psychometrika, 75(3), 474-497. doi: 10.1007/s11336-010-9161-9

Ehm, W. (1991). Binomial approximation to the Poisson binomial distribution. Statistics \& Probability Letters, 11, 7-16.

Fisher, A., Rudin, C., \& Dominici, F. (2019). All models are wrong, but many are useful: Learning a variable's importance by studying an entire class of prediction models simultaneously. Journal of Machine Learning Research, 20.

Fix, E., \& Hodges, J. (1951). Discriminatory analysis, nonparametric discrimination: Consistency properties (Tech. Rep.). Randolph Field, TX: USAF School of Aviation Medicine.

Friedman, J. H. (2003). On multivariate goodness of fit and two sample testing. Proceedings 
of the Conference on Statistical Problems in Particle Physics, Astrophysics, and Cosmology (PHYSTAT2003), 311-313.

Ghosh, R. P., Mallick, B., \& Pourahmadi, M. (2020). Bayesian estimation of correlation matrices of longitudinal data. Bayesian Analysis, 1-20. doi: 10.1214/20-ba1237

Glorot, X., \& Bengio, Y. (2010). Understanding the difficulty of training deep feedforward neural networks. Journal of Machine Learning Research, 9, 249-256.

Goldberg, L. R. (1999). A broad-bandwidth, public domain, personality inventory measuring the lower-level facets of several five-factor models. In I. Meervielde, I. J. Deary, F. De Fruyt, \& F. Ostendorf (Eds.), Personality psychology in europe (Vol. 7, pp. 7-28). Tilburg University Press.

Goodfellow, I., Bengio, Y., \& Courville, A. (2016). Deep learning. Cambridge, MA: MIT Press. Retrieved from http://www. deeplearningbook.org

Gretton, A., Borgwardt, K. M., Rasch, M. J., Schölkopf, B., \& Smola, A. (2012). A kernel two-sample test. Journal of Machine Learning Research, 13, 723-773.

Hastie, T., Tibshirani, R., \& Friedman, J. (2009). The elements of statistical learning: Data mining, inference, and prediction (2nd ed. ed.). New York: Springer. Retrieved from https:// web.stanford.edu/ $\{\sim\}$ hastie/Papers/ESLII.pdf

Hoffman, M. D., Blei, D. M., Wang, C., \& Paisley, J. (2013). Stochastic variational inference. Journal of Machine Learning Research, 14, 1303-1347.

Hooker, G., \& Mentch, L. (2019). Please stop permuting features: An explanation and alternatives. Retrieved from http://arxiv.org/abs/1905.03151

Hu, L. T., \& Bentler, P. M. (1999). Cutoff criteria for fit indexes in covariance structure analysis: Conventional criteria versus new alternatives. Structural Equation Modeling, 6(1), 1-55. doi: 10.1080/10705519909540118

Huber, P., Ronchetti, E., \& Victoria-Feser, M. P. (2004). Estimation of generalized linear latent variable models. Journal of the Royal Statistical Society. Series B: Statistical Methodology, 66(4), 893-908. doi: 10.1111/j.1467-9868.2004.05627.x

Hui, F. K., Warton, D. I., Ormerod, J. T., Haapaniemi, V., \& Taskinen, S. (2017). Variational approximations for generalized linear latent variable models. Journal of Computational and Graphical Statistics, 26(1), 35-43. Retrieved from http://dx.doi .org/10.1080/ 10618600.2016 .1164708 doi: 10.1080/10618600.2016.1164708

Ipsen, N. B., Mattei, P.-A., \& Frellsen, J. (2021). not-MIWAE: Deep generative modelling with missing not at random data. International Conference on Learning Representations (ICLR), 1-18. Retrieved from http://arxiv.org/abs/2006.12871 
Jitkrittum, W., Szabó, Z., Chwialkowski, K., \& Gretton, A. (2016). Interpretable distribution features with maximum testing power. Advances in Neural Information Processing Systems, 181-189.

Jöreskog, K. G., \& Moustaki, I. (2001). Factor analysis of ordinal variables: A comparison of three approaches. Multivariate Behavioral Research, 36(3), 347-387. doi: 10.1207/ S15327906347-387

Kim, I., Ramdas, A., Singh, A., \& Wasserman, L. (2021). Classification accuracy as a proxy for two-sample testing. Annals of Statistics, 49(1), 411-434. doi: 10.1214/20-aos1962

Kingma, D. P., \& Welling, M. (2014). Auto-encoding variational Bayes. International Conference on Learning Representations, 1-14. Retrieved from http://arxiv.org/abs/1312 .6114

Kolmogorov, A. N. (1933). Sulla determinazione empirica di una lgge di distribuzione. Inst. Ital. Attuari,Giorn., 4, 83-91.

Kuhfeld, M. (2019). A posterior predictive model checking method assuming posterior normality for item response theory. Applied Psychological Measurement, 43(2), 125-142. doi: 10 $.1177 / 0146621618779985$

LeCun, Y., Bengio, Y., \& Hinton, G. (2015). Deep learning. Nature Methods, 521(1), 436-444. doi: 10.1038/nmeth.3707

Lee, T., Cai, L., \& Kuhfeld, M. (2016). A poor person's posterior predictive checking of structural equation models. Structural Equation Modeling, 23(2), 206-220. Retrieved from http : / / dx.doi.org/10.1080/10705511.2015.1014041 doi:10.1080/10705511.2015 .1014041

Levy, R., Mislevy, R. J., \& Sinharay, S. (2009). Posterior predictive model checking for multidimensionality in item response theory. Applied Psychological Measurement, 33(7), 519-537. doi: $10.1177 / 0146621608329504$

Liu, Y. (2020). A Riemannian optimization algorithm for joint maximum likelihood estimation of high-dimensional exploratory item factor analysis. Psychometrika, 85(2), 439468. Retrieved from https://doi.org/10.1007/s11336-020-09711-8 doi: $10.1007 / \mathrm{s} 11336-020-09711-8$

Lopez-Paz, D., \& Oquab, M. (2017). Revisiting classifier two-sample tests. 5th International Conference on Learning Representations, ICLR 2017 - Conference Track Proceedings(Section 3), 1-15.

Lundberg, S. M., \& Lee, S. I. (2017). A unified approach to interpreting model predictions. 31st Conference on Neural Information Processing Systems (NIPS 2017), 4766-4775. 
Maccallum, R. C., \& Tucker, L. R. (1991). Representing sources of error in the commonfactor model: Implications for theory and practice. Psychological Bulletin, 109(3), 502-511. Retrieved from file:///Users/Njaal/Dropbox/Library •papers3/ Articles/2004/Unknown/2004-8.pdf \{\\%\} 5Cnpapers3://publication/ uuid/1DF22C4D-FB53-4CF 9-965D-29251B1CB559

Mann, H. B., \& Whitney, D. R. (1947). On a test of whether one of two random variables is stochastically larger than the other. The Annals of Mathematical Statistics, 18(1), 50-60.

Mattei, P.-A., \& Frellsen, J. (2018). MIWAE: Deep generative modelling and imputation of incomplete data. (NeurIPS), 1-5. Retrieved from http: / / arxiv.org/abs/1812.02633

Maydeu-Olivares, A. (2013a). Goodness-of-fit assessment of item response theory models. Measurement, 11(3), 71-101. doi: 10.1080/15366367.2013.831680

Maydeu-Olivares, A. (2013b). Why should we assess the goodness-of-fit of IRT models? Measurement, 11(3), 127-137. doi: 10.1080/15366367.2013.841511

Maydeu-Olivares, A., \& Joe, H. (2005). Limited- And full-information estimation and goodnessof-fit testing in 2n contingency tables: A unified framework. Journal of the American Statistical Association, 100(471), 1009-1020. doi: 10.1198/016214504000002069

Maydeu-Olivares, A., \& Joe, H. (2006). Limited information goodness-of-fit testing in multidimensional contingency tables. Psychometrika, 71(4), 713-732. doi: 10.1007/s11336-005 $-1295-9$

Maydeu-Olivares, A., \& Joe, H. (2014). Assessing approximate fit in categorical data analysis. Multivariate Behavioral Research, 49(4), 305-328. doi: 10.1080/00273171.2014.911075

McKinley, R., \& Reckase, M. (1983). An extension of the two-parameter logistic model to the multidimensional latent space (Tech. Rep.). Iowa City, IA: The American College Testing Program.

Meng, X. L., \& Schilling, S. (1996). Fitting full-information item factor models and an empirical investigation of bridge sampling. Journal of the American Statistical Association, 91(435), 1254-1267. doi: 10.1080/01621459.1996.10476995

Muthén, B. (1978). Contributions to factor analysis of dichotomous variables. Psychometrika, 43(4), 551-560.

Muthén, B. (1984). A general structural equation model with dichotomous, ordered categorical, and continuous latent variable indicators. Psychometrika, 49(1), 115-132.

Natesan, P., Nandakumar, R., Minka, T., \& Rubright, J. D. (2016). Bayesian prior choice in IRT estimation using MCMC and variational Bayes. Frontiers in Psychology, 7(SEP), 1-11. doi: 10.3389/fpsyg.2016.01422 
Pardos, Z. A. (2017). Big data in education and the models that love them. Current Opinion in Behavioral Sciences, 18, 107-113. doi: 10.1016/j.cobeha.2017.11.006

Paszke, A., Gross, S., Chintala, S., Chanan, G., Yang, E., DeVito, Z., ... Lerer, A. (2017). Automatic differentiation in PyTorch. Workshop on Neural Information Processing Systems. doi: $10.1145 / 24680.24681$

Pedregosa, F., Varoquaux, G., Gramfort, A., Michel, V., Thirion, B., Grisel, O., ... Duchesnay, E. (2011). Scikit-learn: Machine learning in Python. Journal of Machine Learning Research, $12,2825-2830$.

Pinheiro, J. C., \& Bates, D. M. (1996). Unconstrained parametrizations for variance-covariance matrices. Statistics and Computing, 6(3), 289-296. doi: 10.1007/BF00140873

Rabe-Hesketh, S., Skrondal, A., \& Pickles, A. (2005). Maximum likelihood estimation of limited and discrete dependent variable models with nested random effects. Journal of Econometrics, 128(2), 301-323. doi: 10.1016/j.jeconom.2004.08.017

Rapisarda, F., Brigo, D., \& Mercurio, F. (2007). Parameterizing correlations: A geometric interpretation. IMA Journal of Management Mathematics, 18(1), 55-73. doi: 10.1093/imaman/ dp1010

Reddi, S. J., Kale, S., \& Kumar, S. (2018). On the convergence of ADAM and beyond. International Conference on Learning Representations. Retrieved from http: / / arxiv.org/ abs/1904.09237

Rezende, D. J., Mohamed, S., \& Wierstra, D. (2014). Stochastic backpropagation and approximate inference in deep generative models. Proceedings of the 31st International Conference on Machine Learning, 32(2), 1278-1286.

Samejima, F. (1969). Estimation of latent ability using a response pattern of graded scores. Psychometrika, 35(1), 139. doi: 10.1007/BF02290599

Schilling, S., \& Bock, R. D. (2005). High-dimensional maximum marginal likelihood item factor analysis by adaptive quadrature. Psychometrika, 70(3), 533-555. doi: 10.1007/s11336-003 $-1141-x$

Sellereite, N., \& Jullum, M. (2020). shapr: An R-package for explaining machine learning models with dependence-aware Shapley values. Journal of Open Source Software, 5(46), 2027. doi: 10.21105/joss.02027

Sinharay, S., Johnson, M. S., \& Stern, H. S. (2006). Posterior predictive assessment of item response theory models. Applied Psychological Measurement, 30(4), 298-321. doi: 10 $.1177 / 0146621605285517$

Smirnov, N. V. (1939). On the estimation of the discrepancy between empirical curves ofdistribution for two independent samples. Bull. Math. Univ. Moscou, 2(2), 3-14. 
Song, X. Y., \& Lee, S. Y. (2005). A multivariate probit latent variable model for analyzing dichotomous responses. Statistica Sinica, 15(3), 645-664.

Steiger, J. H. (1980). Statistically based tests for the number of common factors. In The annual meeting of the psychometric society. Iowa City, IA.

Student. (1908). The probable error of a mean. Biometrika, 6(1), 1-25.

Tang, D., \& Ranganath, R. (2019). The variational predictive natural gradient. 36th International Conference on Machine Learning, ICML 2019, 2019-June, 10735-10744.

Thissen, D., \& Steinberg, L. (1997). A response model for multiple-choice items. In W. J. van der Linden \& R. K. Hambleton (Eds.), Handbook of modern item response theory (pp. 51-66). New York, NY: Springer-Verlag.

Tsay, R. S., \& Pourahmadi, M. (2017). Modelling structured correlation matrices. Biometrika, 104(1), 237-242. doi: 10.1093/biomet/asw061

Tucker, G., Maddison, C. J., Lawson, D., \& Gu, S. (2019). Doubly reparameterized gradient estimators for Monte Carlo objectives. 7th International Conference on Learning Representations, ICLR 2019(2015), 1-14.

Tucker, L. R., \& Lewis, C. (1973). A reliability coefficient for maximum likelihood factor analysis. Psychometrika, 38(1), 1-10.

Urban, C. J., \& Bauer, D. J. (2021). A deep learning algorithm for high-dimensional exploratory item factor analysis. Psychometrika, 86(1), 1-29. Retrieved from https://doi.org/ $10.1007 /$ s11336-021-09748-3 doi: 10.1007/s11336-021-09748-3

Wilcoxon, F. (1945). Individual comparisons of grouped data by ranking methods. Biometrics Bulletin, 1(6), 80-83. doi: 10.1093/jee/39.2.269

Wirth, R. J., \& Edwards, M. C. (2007). Item factor analysis: Current approaches and future directions. Psychological Methods, 12(1), 58-79. doi: 10.1037/1082-989X.12.1.58

Woo, S. E., Tay, L., \& Proctor, R. W. (Eds.). (2020). Big data in psychological research. American Psychological Association. doi: https://doi.org/10.1037/0000193-000

Wu, M., Davis, R. L., Domingue, B. W., Piech, C., \& Goodman, N. (2020). Variational item response theory : Fast, accurate, and expressive.

Yarkoni, T., \& Westfall, J. (2017). Choosing prediction over explanation in psychology: Lessons from machine learning. Perspectives on Psychological Science, 12(6), 1100-1122. doi: $10.1177 / 1745691617693393$

Yun, J., Lozano, A. C., \& Yang, E. (2020). A general family of stochastic proximal gradient methods for deep learning. Retrieved from http://arxiv.org/abs/2007.07484 
Zhang, C., Butepage, J., Kjellstrom, H., \& Mandt, S. (2019). Advances in variational inference. IEEE Transactions on Pattern Analysis and Machine Intelligence, 41(8), 2008-2026. doi: 10.1109/TPAMI.2018.2889774

Zhang, S., Chen, Y., \& Liu, Y. (2020). An improved stochastic EM algorithm for large-scale full-information item factor analysis. British Journal of Mathematical and Statistical Psychology, 73(1), 44-71. doi: 10.1111/bmsp.12153 